\title{
Comparative study of normal and branched alkane monolayer films adsorbed on a solid surface. II. Dynamics
}

\author{
A. D. Enevoldsen and F. Y. Hansen ${ }^{\text {a) }}$ \\ Department of Chemistry, Technical University of Denmark, IK 207 DTU, DK-2800 Lyngby, Denmark
}

\author{
A. Diama and H. Taub \\ Department of Physics and Astronomy, University of Missouri-Columbia, Columbia, Missouri 65211 \\ and University of Missouri Research Reactor Center, University of Missouri-Columbia, Columbia, \\ Missouri 65211 \\ R. M. Dimeo, D. A. Neumann, and J. R. D. Copley \\ Center for Neutron Research, National Institute of Standards and Technology, Gaithersburg, \\ Maryland 20899-8562
}

(Received 21 September 2006; accepted 9 January 2007; published online 13 March 2007)

\begin{abstract}
The dynamics of monolayer films of the $n$-alkane tetracosane $\left(n-\mathrm{C}_{24} \mathrm{H}_{52}\right)$ and the branched alkane squalane $\left(\mathrm{C}_{30} \mathrm{H}_{62}\right)$ adsorbed on graphite have been studied by quasielastic and inelastic neutron scattering and molecular dynamics (MD) simulations. Both molecules have 24 carbon atoms along their carbon backbone, and squalane has an additional six methyl side groups symmetrically placed along its length. The authors' principal objective has been to determine the influence of the side groups on the dynamics of the squalane monolayer and thereby assess its potential as a nanoscale lubricant. To investigate the dynamics of these monolayers they used both the disk chopper spectrometer (DCS) and the high flux backscattering spectrometer (HFBS) at the National Institute of Standards and Technology. These instruments made it possible to study dynamical processes such as molecular diffusive motions and vibrations on very different time scales: 1-40 ps (DCS) and 0.1-4 ns (HFBS). The MD simulations were done on corresponding time scales and were used to interpret the neutron spectra. The authors found that the dynamics of the two monolayers are qualitatively similar on the respective time scales and that there are only small quantitative differences that can be understood in terms of the different masses and moments of inertia of the two molecules. In the course of this study, the authors developed a procedure to separate out the low-frequency vibrational modes in the spectra, thereby facilitating an analysis of the quasielastic scattering. They conclude that there are no major differences in the monolayer dynamics caused by intramolecular branching. It remains to be seen whether this similarity in monolayer dynamics also holds for the lubricating properties of these molecules in confined geometries. (C) 2007 American Institute of Physics. [DOI: 10.1063/1.2464092]
\end{abstract}

\section{INTRODUCTION}

In a preceding paper, ${ }^{1}$ we reported a detailed experimental and molecular dynamics study of the structures of monolayer films of two intermediate-length alkanes adsorbed on graphite. One was the $n$-alkane tetracosane, $\mathrm{C}_{24} \mathrm{H}_{50}(\mathrm{C} 24)$, and the other the branched alkane squalane, $\mathrm{C}_{30} \mathrm{H}_{62}$. Both have a backbone of 24 carbon atoms, and squalane has an additional six methyl side groups symmetrically placed along the chain. The principal issue in that study was to compare the structures of the monolayer films formed by the two molecules when adsorbed on a solid surface. Although the lengths of the molecules are identical, bulk properties such as the melting points are quite different. Squalane has a bulk melting point of $234 \mathrm{~K}, 88 \mathrm{~K}$ below that of $\mathrm{C} 24$. This difference is caused by the methyl side groups in the squalane molecule. The low melting point of squalane is probably the main reason why it is a better lubricant at room

${ }^{a)}$ Electronic mail: flemming @kemi.dtu.dk temperature, ${ }^{2,3}$ it is a fluid at this temperature, whereas $\mathrm{C} 24$ is a wax. However, it is not obvious that bulk characteristics alone determine the lubrication properties of a material. Usually, a lubricant is distributed in a thin film between two solid bodies in relative motion; and, in certain modern electromechanical devices, this film thickness may reach nanoscale dimensions with room for only a very few layers of molecules. In Ref. 1, we found that a graphite surface stabilized a monolayer film of squalane, resulting in a melting point of $325 \mathrm{~K}$ which is only about $15 \mathrm{~K}$ below that of a C24 monolayer on graphite. Thus, because both films are solid at room temperature, it is unlikely that there will be a difference in their lubricating properties at the nanoscale.

In this paper, we complement our structural studies of squalane and $\mathrm{C} 24$ monolayers by an extensive investigation of their dynamics. We have used inelastic neutron scattering to study both the diffusive and vibratory modes in monolayer films of the two molecules and have conducted extensive molecular dynamics (MD) simulations in order to interpret 
the observed spectra. By combining the experimental studies and MD simulations, we gain a detailed understanding of the monolayer dynamics.

It is characteristic of systems of flexible chainlike molecules such as the alkanes that their excitations have a rather broad power spectrum extending upward from zero frequency. For alkanes, their spectral width is about $40-50 \mathrm{meV}$, excluding the high frequency bond-stretching modes but including angle bends and dihedral torsions. In MD simulations, we may select the time scale of the excitations to be studied by a proper choice of both the time step and the maximum time used in the calculation of the relevant time correlation functions. These characteristic times correspond to an energy range, or a dynamic range, and an energy resolution, respectively, and it is these parameters that also characterize the spectrometers selected for the experiments. In this study, we have used both the disk chopper spectrometer (DCS) and the high flux backscattering spectrometer (HFBS) at the Center for Neutron Research at the National Institute of Standards and Technology. These instruments allowed us to probe dynamical processes on a time scale of 1-40 ps and 0.1-4 ns, respectively. By obtaining spectra and conducting MD simulations at different temperatures and wave vector transfers, we are able to investigate the monolayer dynamics as its structure changes. For example, we have used this technique to determine the role of gauche defects in driving the melting transition in monolayer alkane films. ${ }^{4}$

This paper is organized as follows. In Sec. II, we review the basic features of the scattering functions and then we give examples of processes occurring on different time scales. In Sec. III, we summarize the method of the MD simulations briefly since it has already been described previously. In Sec. IV, we describe the procedure used to fit the observed spectra to a model of elastic and quasielastic scattering. The fit to the DCS spectra presented a particular problem because the quasielastic and inelastic regions of the spectra overlap. Therefore, it is difficult to obtain a unique fit in these regions; we have proposed a procedure to minimize the effect of their overlap. In Sec. V, we use MD simulations to identify features in the inelastic spectra. In Sec. VI, we present and discuss the quasielastic spectra. A summary and conclusions are given in Sec. VII.

\section{SCATTERING FUNCTIONS}

In a neutron scattering experiment, the coherent and incoherent scattered intensities are proportional to the scattering functions $S_{\text {coh }}(\mathbf{q}, \omega)$ and $S_{\text {inc }}(\mathbf{q}, \omega)$, respectively. ${ }^{5}$ Here, $\mathbf{q}$ is the neutron wave vector transfer and $\hbar \omega$ is the energy exchange between neutrons and the sample. In the experiments reported here, we use protonated alkanes, which means that the scattered intensity is completely dominated by the large incoherent scattering of hydrogen.

From a MD simulation, we may calculate the scattering functions and thereby make direct contact to experiments. The scattering functions are the space and time Fourier trans- forms of the dynamic pair correlation functions $G(\mathbf{r}, t)$ (coherent scattering) and $G_{s}(\mathbf{r}, t)$ (incoherent scattering). $G(\mathbf{r}, t)$ may be written as a sum of two terms,

$$
G(\mathbf{r}, t)=G_{s}(\mathbf{r}, t)+G_{d}(\mathbf{r}, t),
$$

with

$$
\begin{aligned}
G_{s}(\mathbf{r}, t) & =\frac{1}{N} \sum_{j=1}^{N}\left\langle\delta\left(\mathbf{r}-\left(\mathbf{R}_{j}(t)-\mathbf{R}_{j}(0)\right)\right)\right\rangle, \\
G_{d}(\mathbf{r}, t) & =\frac{1}{N} \sum_{j \neq i}^{N}\left\langle\delta\left(\mathbf{r}-\left(\mathbf{R}_{j}(t)-\mathbf{R}_{i}(0)\right)\right)\right\rangle .
\end{aligned}
$$

The brackets indicate an ensemble average and $G_{s}(\mathbf{r}, t)$ gives the probability of finding an atom $j$ at $\mathbf{R}_{j}(t)$ at time $t$, given that the same atom was at $\mathbf{R}_{j}(0)$ at time zero. $G_{d}(\mathbf{r}, t)$ gives the probability of finding an atom $j$ at $\mathbf{R}_{j}(t)$ at time $t$, given that a different atom $i$ was at $\mathbf{R}_{i}(0)$ at time zero. $G(\mathbf{r}, t)$, the sum of $G_{s}(\mathbf{r}, t)$ and $G_{d}(\mathbf{r}, t)$, therefore gives the probability of finding an atom $j$ at $\mathbf{R}_{j}(r, t)$, given that any atom, the same or a different one, was at $\mathbf{R}_{i}(0)$ at time zero.

In a simulation, it is inconvenient to determine the dynamic pair correlation functions directly since they are given in terms of delta functions which are not ordinary continuous functions. It is more convenient to calculate the space Fourier transforms of the functions called the intermediate scattering functions. They are found to be

$$
\begin{aligned}
& F_{s}(\mathbf{q}, t)=\frac{1}{N} \sum_{j=1}^{N}\left\langle\exp \left(i \mathbf{q} \cdot\left(\mathbf{R}_{j}(t)-\mathbf{R}_{j}(0)\right)\right)\right\rangle, \\
& F_{d}(\mathbf{q}, t)=\frac{1}{N} \sum_{j \neq i}^{N}\left\langle\exp \left(i \mathbf{q} \cdot\left(\mathbf{R}_{j}(t)-\mathbf{R}_{i}(0)\right)\right)\right\rangle,
\end{aligned}
$$

and they are continuous functions which are easy to determine from a simulation.

Since we are going to consider the incoherent scattering in this work, we limit our discussion to the incoherent scattering function or the self-scattering function. Because neutrons interact very weakly with the sample, we need to have as much material as possible in the beam; this is achieved by using a high-surface-area polycrystalline substrate, Grafoil. The scattering will therefore give a powder average rather than a single-crystal spectrum. In general, this requires performing a three-dimensional average in the first of Eqs. (3) over all orientations of $\mathbf{R}_{j}(t)-\mathbf{R}_{j}(0)$ with respect to $\mathbf{q}$ that is fixed in space. However, there is some preferred orientation of the graphite crystallites in the Grafoil sheets such that the majority of them lie with their basal-plane surfaces within $\pm 30^{\circ}$ of the scattering plane. ${ }^{6}$ For this reason, we have used a two-dimensional rather than a three dimensional powder average of the scattering function given by 


$$
\begin{aligned}
F_{s}(q, t)= & \frac{1}{2 \pi} \int_{0}^{2 \pi} \mathrm{d} \phi \sum_{j=1}^{N}\left\langle\exp \left(i \mathbf{q} \cdot\left(\mathbf{R}_{j}(t)-\mathbf{R}_{j}(0)\right)\right)\right\rangle \\
= & \frac{1}{2 \pi} \int_{0}^{2 \pi} \mathrm{d} \phi \sum_{j=1}^{N}\left\langle\exp \left(i|\mathbf{q}|\left|\mathbf{R}_{j}(t)-\mathbf{R}_{j}(0)\right| \cos \phi\right)\right\rangle \\
= & \frac{1}{2 \pi} \int_{0}^{2 \pi} \mathrm{d} \phi \sum_{j=1}^{N}\left[\left\langle\cos \left(i|\mathbf{q}|\left|\mathbf{R}_{j}(t)-\mathbf{R}_{j}(0)\right| \cos \phi\right)\right.\right. \\
& \left.\left.+i \sin \left(|\mathbf{q}|\left|\mathbf{R}_{j}(t)-\mathbf{R}_{j}(0)\right|\right) \cos \phi\right\rangle\right] \\
= & \sum_{j=1}^{N}\left\langle J_{0}\left(q\left[R_{j}(t)-R_{j}(0)\right]\right)\right\rangle
\end{aligned}
$$

where $J_{0}(x)$ is the zero order Bessel function of the first kind. ${ }^{7}$ The intermediate scattering function is equal to unity at time zero and decreases with time. To make contact with experiment, which measures the scattering function, we need to calculate the time Fourier transform of the intermediate scattering function, that is,

$$
S_{s}(q, \omega)=S_{\mathrm{inc}}(q, \omega)=\int_{-\infty}^{\infty} \mathrm{d} t \exp (i \omega t) F_{s}(q, t)
$$

Alternatively, we may determine the intermediate scattering function from the experimental scattering function by a frequency Fourier transform of the scattering function. We have done both, ${ }^{8}$ but, in this paper, we Fourier-transform the MD results.

\section{A. Scattering functions and time scales}

In order to facilitate our later discussion, we now give a qualitative description of the scattering functions for processes relevant to this study.

We begin with the dynamical pair correlation function $G_{s}(r, t)$ defined as a probability density such that $G_{s}(r, t) \mathrm{d} r$ is the probability that an atom has moved the distance $r, r+\mathrm{d} r$ in time $t$. At time zero, $G_{s}(r, t)$ has the shape of a delta function at $r=0$ for all processes; at later times, we sketch in Fig. 1(a) its behavior for processes occurring on different time scales. As time evolves, we see a broadening of $G_{s}(r, t)$, indicating that the atom under consideration may have moved away from its initial position, and we monitor $G_{s}(r, t)$ with a certain time resolution. In a MD simulation, the greatest time resolution achievable corresponds to the integration time step $\mathrm{d} t$. This time step will usually be too small for the processes of interest so that the correlation function is instead monitored every $n$ integration time steps, corresponding to a time resolution of $t_{\min }=n \mathrm{~d} t \mathrm{~s}$. For slow processes we choose a large $n$, and for fast processes a small $n$. The choice of $t_{\min }$ also sets the maximum frequency $\nu_{\max }$ of an excitation in the system that we will be able to study, since $\nu_{\max }$ $=1 /\left(2 t_{\min }\right)$. The factor of 2 in the denominator appears because both positive and negative frequencies are included in the time Fourier transform. We continue the simulation for some finite time, $t_{\max }$, the time window, that determines the energy, or frequency, resolution in the simulation. That is, the lowest frequency that we will be able to monitor is given by
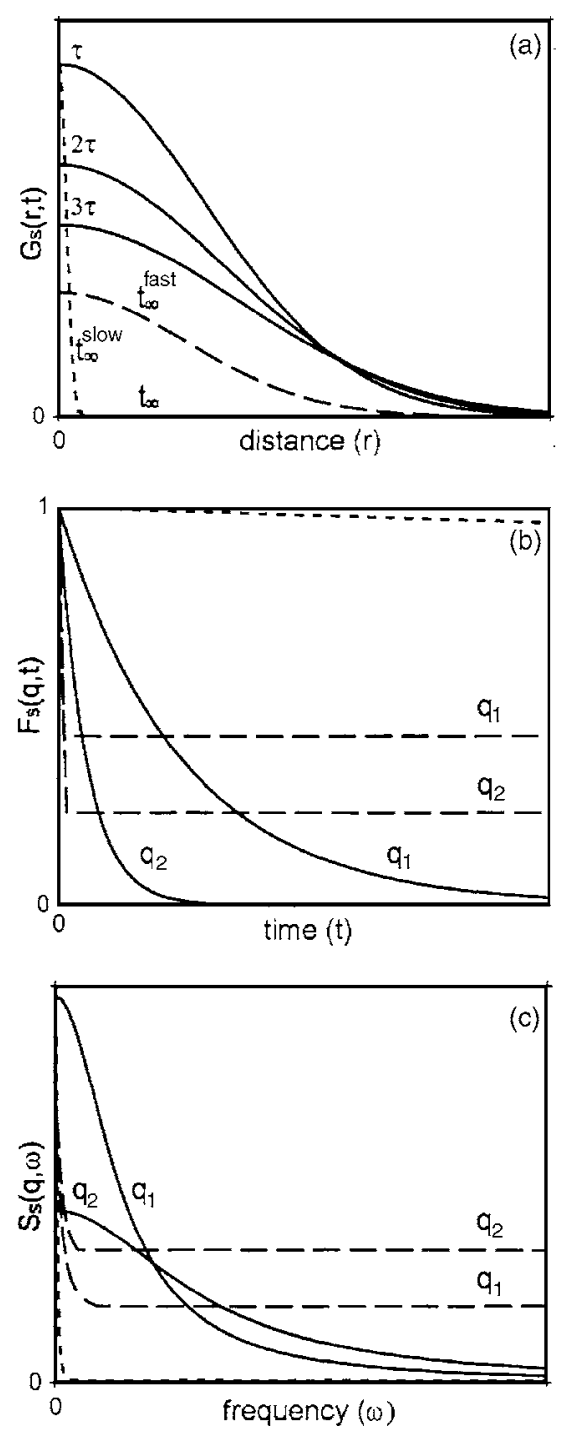

FIG. 1. (a) Sketch of the dynamical pair correlation function $G_{s}(r, t)$; (b) intermediate scattering function $F_{s}(q, t)$; and (c) scattering function $S_{s}(q, \omega)$ for dynamical processes occurring on different time scales. The solid curves represent the functions for a process with a time scale matched to the time/ energy resolution and dynamic range of our experiments and simulations. The long-dashed curves are for a fast process and the short-dashed curves are for a slow process relative to the chosen time resolution. Note that $\tau$ $=t_{\min }$ (Table I) and that in (b) and (c), $q_{2}>q_{1}$.

$\Delta \nu=1 / t_{\max }$. In experiments, the energy resolution and energy transfer range accessible will depend on the spectrometer.

Let us now assume that we monitor a dynamical process at a time interval of $t_{\min }$ seconds. Then, the first dynamical pair correlation function we monitor after $t=0$ is the one labeled $\tau=t_{\min }$ in Fig. 1(a), followed by the ones at $2 \tau, 3 \tau$, etc. until we reach the limiting line shape at the bottom of Fig. 1(a) labeled $t_{\infty}$, corresponding to $t_{\max }$. This time resolution is seen to be adequate since the dynamical pair correlation functions drawn with solid lines evolve smoothly toward their limiting shape.

The long-dashed curve in Fig. 1(a) represents the pair correlation function for a fast process for which the limiting shape is reached before or at the first time $\tau=t_{\min }$ that we monitor the correlation function. We are not able to follow the evolution of the correlation function earlier because the 
process is too fast with respect to the chosen time resolution. It would be necessary to choose a finer time resolution in order to study the time evolution of that correlation function. Similarly, in an experiment, we would need to choose a larger energy transfer range to study the time evolution of that process.

The short-dashed curve in Fig. 1(a) corresponds to a very slow process. At infinite time, that is, $t_{\max }$, the atom has hardly moved away from the start position, as illustrated by the very narrow dynamical pair correlation function. Note that the symbols $t_{\infty}^{\text {slow }}$ and $t_{\infty}^{\text {fast }}$ refer to the same time, $t_{\max }$, with the superscripts differentiating between the slow and fast processes.

There are three characteristic shapes for the limiting dynamical pair correlation function, independent of the time scale of the process. For the process in Fig. 1(a) that has been used to set the time resolution in our simulations (solid lines), we have shown a limiting dynamical pair correlation function labeled $t_{\infty}$, which is almost zero and nearly constant over the entire $r$ range. Physically, this behavior means that the atom may be anywhere in space with an infinitesimally small probability of being at a specific position. A good example of such a process is the translational diffusive motion of an atom in a fluid. Another characteristic shape of $G_{s}(r, t)$ is illustrated for the fast process (long-dashed line) where the atom never moves very far away from its initial position, so the dynamical pair correlation function remains finite in the neighborhood of its starting point. Good examples of such a process are the vibrational motion of the atoms in a crystal and atoms in a rotational motion. A third characteristic shape of $G_{s}(r, t)$ is illustrated by the slow process (short-dashed line) where the atom hardly moves in the time window of interest. An example of this may also be an atom in a slow translational or rotational motion or in a slow motion associated with conformational changes of the molecule.

We next study the form of the intermediate scattering function for the three characteristic shapes of $G_{s}(r, t)$ in Fig. 1(a) that we have described above. We write $G_{s}(r, t)$ in the following way:

$G_{s}(r, t)=G_{s}(r, \infty)+G_{s}^{\prime}(r, t) \quad$ with $G_{s}^{\prime}(r, t) \rightarrow 0$ for $t \rightarrow \infty$,

i.e. as a sum of the limiting function as $t \rightarrow \infty$ and of a timedependent function that approaches zero at infinite time. Then, taking the space Fourier transform, we find the intermediate scattering function

$F_{s}(q, t)=F_{s}(q, \infty)+F_{s}^{\prime}(q, t) \quad$ with $F_{s}^{\prime}(q, t) \rightarrow 0$ for $t \rightarrow \infty$.

The intermediate scattering functions corresponding to the three characteristic shapes of $G_{s}(r, t)$ in Fig. 1(a) are sketched in Fig. 1(b). At time zero, the intermediate scattering functions are equal to unity according to Eq. (4) in all three cases.

The intermediate scattering function for the process to which the selected time resolution has been matched (solid curves) has a smooth decay toward its limiting value of zero as determined by $F_{s}^{\prime}(q, t)$ toward its limiting value of zero. In this case, we have assumed $G_{s}(r, \infty)=0$ and hence $F_{s}(q, \infty)$ $=0$. As shown by the two solid curves, the decay rate of $F_{s}(q, t)$ depends on the wave vector transfer $q$. Had the atomic motions in the process been restricted to the neighborhood of the initial position, then we would have seen a decay to a nonzero limiting value, as shown for the fast mode (long-dashed curves) for two different wave vector transfers $q_{1}$ and $q_{2}$. We emphasize that whether or not the intermediate scattering function decays to a zero or a nonzero limiting value is independent of the time scale of the process and is determined solely by the spatial extent of the diffusive motion at infinite time as given by $G_{s}(r, \infty)$.

For the fast process (long-dashed curves) we see an almost instantaneous drop of $F_{s}(q, t)$ to a constant nonzero level. The approach of $F_{s}(q, t)$ to its asymptotic value is governed by $F_{s}^{\prime}(q, t)$, which depends on the time scale of the process; in this case, it is almost a delta function around time zero. The constant nonzero level at long times is determined by the space Fourier transform of the limiting dynamical pair correlation function and is therefore a function of $q$ as determined by the range of $r$ contributing to the integral. At small $q$, there will be contributions from a large region of $r$ and therefore from most of the limiting dynamical pair correlation function. The level will therefore be at a high value, although still less than unity. When $q$ increases, the range of $r$ values that contribute significantly to the intermediate scattering function is reduced, so there will only be nonzero contributions to the Fourier integral from the small $r$ part of the dynamical pair correlation function. The level at large times is therefore lower for $q_{2}$ than for $q_{1}$, if $q_{2}>q_{1}$ in Fig. 1(b).

For the slow process, the intermediate scattering function is the space Fourier transform of a near-delta function in space giving an almost constant value close to unity in the entire time window [short-dashed curve in Fig. 1(b)]. For clarity, we have omitted showing a $q$ dependence of the intermediate scattering function for the slow process.

Finally, let us look at the scattering function given by

$$
S_{s}(q, \omega)=2 \pi F_{s}(q, \infty) \delta(\omega)+S_{s}^{\prime}(q, \omega) .
$$

We see from this equation that there may be two contributions to the scattering functions, an elastic contribution represented by the first term on the right hand side and a frequency-dependent term. The scattering functions for the processes studied above are sketched in Fig. 1(c).

The process to which the selected time resolution was matched will have a frequency and wave vector dependent line shape, as shown by the solid curves in Fig. 1(c) that will be characteristic of the type of process involved. In this example, there will be no elastic contribution because the limiting value of the dynamical pair correlation function dropped to zero; had it not done so, there would also be an elastic contribution.

The intermediate scattering function for the fast process in Fig. 1(b) consists of two parts: an abrupt decay of $F_{s}(q, t)$ from unity at $t=0$, which we can consider to be a delta function in time, and a constant time-independent part for $t>0$. The time Fourier transform of a delta function is a constant (independent of the frequency) and that of the time- 
independent part of $F_{s}(q, t)$ is a delta function in the frequency. Thus, the scattering function for the fast process is composed of a delta function in frequency $\omega$ and a smooth, frequency-independent part, as sketched in Fig. 1(c). Thus, for a fast process one will observe a smooth frequencyindependent intensity that will be part of the background intensity and a contribution to the elastic intensity.

The slow process will have a shape like a delta function at zero frequency, as sketched in Fig. 1(c), since it is the time Fourier transform of a nearly constant intermediate scattering function. In an experiment, it may be hidden in the elastic scattering, if the energy resolution is insufficient. In order to be able to monitor the slow process in a simulation, we should extend the time window to longer times $t_{\max }$, long enough for the intermediate scattering function to change significantly. The energy resolution will then be sufficient to distinguish the scattering function from the elastic scattering.

\section{B. Line shape of scattering functions for simple types of motion}

The line shape of the scattering functions in Fig. 1 depends on the type of motion involved. It is possible to derive analytical expressions for some simple types of motion, such as translational and rotational diffusion. These expressions are useful as benchmarks, even when the translational and rotational modes are not simple but coupled in a complicated way.

\section{Continuous translational diffusion}

For simple translational diffusion, ${ }^{5,9}$ we find from a description based on the Langevin equation that the dynamical pair correlation function is a Gaussian in $r$ with a timedependent width, and the intermediate scattering function will accordingly be an exponentially decaying function. This results in a Lorentzian line shape for the scattering function, given by

$$
S_{s}(q, \omega)=S_{\mathrm{inc}}=\frac{A}{\pi} \frac{D q^{2}}{\omega^{2}+\left(D q^{2}\right)^{2}},
$$

where $D$ is the diffusion constant and $A$ the intensity. The half-width at half maximum (HWHM) of the Lorentzian is given by

$$
\mathrm{HWHM}=D q^{2},
$$

so that there is a linear relationship between the HWHM and $q^{2}$ when the process is a simple continuous random walk. The diffusion constant $D$ determines the time scale of the diffusion process. A large $D$ leads to a fast process. Other more complicated forms of translational diffusion have also been studied. ${ }^{9}$ These include oscillating diffusive motion where the molecule, after a period of translational diffusion, may be trapped for a while, performing an oscillatory motion before resuming a diffusive motion. The $q$ dependence of the HWHM of the scattering function for that kind of motion is the same as the one for translational continuous diffusion. However, the interpretation of the coefficient of $q^{2}$ is more complex in these more general cases.

\section{Rotational diffusion}

Following Bee ${ }^{10}$ the scattering function for a continuous uniaxial rotation is

$$
S(q, \omega)=J_{0}^{2}(q r) \delta(\omega)+2 \sum_{m=1}^{\infty} J_{m}^{2}(q r) \frac{1}{\pi} \frac{D_{r} m^{2}}{\omega^{2}+\left(D_{r} m^{2}\right)^{2}}
$$

Here, we have set $\theta=90^{\circ}$ in the expression in Bee's book, ${ }^{10}$ corresponding to having the scattering vector $q$ in the plane of the monolayer as in our experiments. $J_{m}$ is the $m$ th cylindrical Bessel function, ${ }^{7} r$ the distance of the atoms producing the scattering from the axis of rotation, and $D_{r}$ the rotational diffusion constant. The expression consists of an elastic part, $J_{0}^{2}(q r)$, which represents the constant value of the intermediate scattering function at large times and a quasielastic term, here written as a sum of $q$-independent Lorentzians with $\mathrm{HWHM}=D_{r} m^{2}$. The $q$-dependent Bessel functions in the sum determine the intensity of the quasielastic scattering, and for a given value of the argument $q r$, the value of the Bessel functions decreases with increasing $m$. Hence, the $J_{1}$ function will dominate the scattering function, and the rotational diffusion will be characterized by a HWHM that is independent of the wave vector $q$ and an intensity given by the $J_{1}^{2}(q r)$ Bessel function.

Finally, we note that we use the pseudoatom model for the alkanes where the methyl and methylene groups have been replaced by pseudoatoms of their respective mass at the $\mathrm{C}$ atom positions. The principal moments of inertia of the molecules are then different from those of the real molecule. This effect is particularly important for rotation around the long molecular axis, for which the moment of inertia is about half of its true value. The error in the other moments of inertia is smaller and is in the $10 \%$ range. This means that in the simulations the rotational motion about the long axis of the C24 molecule may be $\sqrt{2}$ times faster than in the real molecule, resulting in either a HWHM that is too large or a diffusive process so fast that the corresponding quasielastic intensity ends up in the background.

\section{MOLECULAR DYNAMICS SIMULATIONS}

We have used the same setup procedure as in our simulation studies of the $\mathrm{C} 24$ and squalane monolayer structures. ${ }^{1}$ The systems were equilibrated for 1-2 ns depending on the temperature before generating data for the calculation of the relevant time correlation functions. Since a variety of time correlation functions will often be of relevance, it is more convenient to calculate them separately rather than simultaneously with the simulations. This requires the generation of data files along the MD trajectory containing the relevant properties such as atomic positions and velocities at regular time intervals.

Suppose we are interested in some property $A(t)$, which is a function of the atomic positions and velocities. From the data in the generated data file, $A(t)$ will be available at equidistant intervals of time $\tau=t_{\text {min }}$; typically, $\tau$ will be some multiple of the time step in the numerical integration of the equations of motion. Whether it is a small or large multiple depends on the process. We write the time average $C(t)$ of the non-normalized autocorrelation function of $A$ as 
TABLE I. Time and energy resolutions in experiments and simulations.

\begin{tabular}{lccccc}
\hline \hline & \multicolumn{2}{c}{ Expt. } & & \multicolumn{2}{c}{ Simulation } \\
\cline { 2 - 3 } \cline { 5 - 6 } \cline { 5 - 6 } & DCS & HFBS & & DCS & HFBS \\
\hline$t_{\min }(\mathrm{ps})$ & 0.413 & 115 & & 1 & 12 \\
$t_{\max }(\mathrm{ps})$ & 41.3 & 4130 & & 150 & 2400 \\
$\Delta \nu(\mu \mathrm{eV})$ & 100.0 & 1.0 & & 27.6 & 1.72 \\
$\Delta \nu(\mathrm{THz})$ & $2.4 \times 10^{-2}$ & $2.4 \times 10^{-4}$ & & $6.67 \times 10^{-3}$ & $4.17 \times 10^{-4}$ \\
$\nu_{\max }(\mu \mathrm{eV})$ & 10000.0 & 36.0 & & 4130.0 & 345.0 \\
$\nu_{\max }(\mathrm{THz})$ & 2.42 & $8.71 \times 10^{-3}$ & & 1.0 & $8.33 \times 10^{-2}$ \\
\hline \hline
\end{tabular}

$$
C(t)=\langle A(t) A(0)\rangle=\frac{1}{n_{\max } \tau} \sum_{n=1}^{n_{\max }} A(n \tau) A(n \tau+t) .
$$

That is, we average the product of $A$ at time origin $n \tau$ and $A$ at the time $t$ later over $n_{\max }$ choices of time origins in the data time sequence. Clearly, for each value of $t$, the value of $n \tau+t$ must never exceed the maximum time $n_{\text {run }} \tau=t_{\text {max }}$ corresponding to the maximum number of data sets, $n_{\text {run }}$, stored in the data file. This implies that the short-time correlations, corresponding to small $t$, will be determined with greater statistical precision than long time correlations because the number of terms in the average, $n_{\max }$, will be larger for shorter times. Therefore, it is customary to limit the calculation of the time correlation functions to shorter times, typically to times equal to $(0.5-0.75) t_{\max }$, or alternatively to calculate the correlation function for larger times than required. As expressed in Eq. (12), each successive data set in the data file is used as a time origin. In practice, this is not necessary, since successive origins are usually highly correlated. We achieve a faster calculation by summing over every $n_{\text {gap }}$ th data set as the time origin, with $n_{\text {gap }}$ typically $5-10$.

Since we are interested in self- or single-particle time correlation functions, we may improve the statistics of the results by also including an average over all $N$ atoms in the simulation; i.e.,

$$
C(t)=\frac{1}{N} \sum_{i=1}^{N}\left\langle A_{i}(t) A_{i}(0)\right\rangle=\frac{1}{N n_{\max } \tau} \sum_{i=1}^{N} \sum_{n=1}^{n_{\max }} A_{i}(n \tau) A_{i}(n \tau+t) .
$$

Table I summarizes the energy and time resolutions in the experiments and the simulations. The parameters for the simulations were chosen as a compromise to give reasonable computation times.

There is a practical problem with the calculation of the scattering functions $S_{s}(q, \omega)$ from the intermediate scattering functions $F_{s}(q, t)$ if they have not converged to zero in the time window used in the calculation of the time correlation functions. This will be the case when rotational and intramolecular motions are considered because the intermediate scattering function then levels out at a nonzero value, as illustrated in Fig. 1(b). If we calculate the time Fourier transform of such a function directly, then the scattering function will be so noisy around the zero frequency that it is impossible to extract the HWHM of the quasielastic line. Therefore, it is important to subtract the constant level from the intermediate scattering function first before calculating the time Fourier transform. This corresponds to a calculation of the time Fourier transform of the time-dependent part, $F_{s}^{\prime}(q, t)$, in Eq. (7). In situations where the intermediate scattering function has not quite leveled out within the given time window, the background subtraction becomes uncertain. This may also produce noise in the resulting scattering function and may cause problems in the determination of the HWHM of the quasielastic line.

Similarly, the time Fourier transform of the intermediate scattering function for a fast process as sketched in Fig. 1(b) (long-dashed curves) should give flat featureless scattering functions [Fig. 1(c)] that add intensity to the background. In practice, numerical problems produce a rather flat scattering function that starts out with a low value at small frequencies and slowly increases to a broad maximum at nonzero frequency and then decays toward zero at high frequency. It is easy to distinguish such line shape from a proper one, as sketched in Fig. 1(c) (solid curves) for which the time resolution in the simulations is adequate.

Finally, we have developed a projection operator method $^{11}$ that allows us to project out the translational, rotational, and intramolecular motions of a molecule. This has proved very useful in analyzing the dynamics of complicated molecules, as will be discussed below.

\section{EXPERIMENTS}

The quasielastic neutron scattering experiments were carried out on the disk chopper spectrometer ${ }^{13-15}$ (DCS) and on the high flux backscattering spectrometer ${ }^{16,17}$ (HFBS) at the National Institute of Standards and Technology Center for Neutron Research. The design and principle of operation of the two instruments are different, and this results in their very different energy resolution and dynamic range. The HFBS instrument has a relatively high energy resolution of $\sim 1 \mu \mathrm{eV}$ and a somewhat limited dynamic range of $\sim 36 \mu \mathrm{eV}$ (Ref. 13), whereas we use the DCS instrument in a mode with a lower energy resolution of $\sim 100 \mu \mathrm{eV}$ and a much larger dynamic range of $10 \mathrm{meV}$. In this way, the HFBS and DCS allow the probing of dynamical processes on time scales of $0.1-4 \mathrm{~ns}$ and 1-40 ps, respectively.

The samples for both spectrometers were prepared by depositing C24 and squalane from their vapor phase onto an exfoliated graphite substrate (Grafoil) inside a stainless steel cell, as described previously. ${ }^{1,18}$ Prior to deposition, the Grafoil substrate was cleaned by heating in vacuum, and then its surface area was determined using a nitrogen vapor pressure isotherm at $77 \mathrm{~K} .{ }^{6}$ The $\mathrm{C} 24$ and squalane both had a purity of $99 \%$ and were used as supplied. After deposition, the Grafoil disks were transferred under an inert atmosphere to an aluminum sample cell sealed with indium $\mathrm{O}$ rings suitable for the temperature range of the quasielastic neutron scattering measurements, $100-400 \mathrm{~K}$. To reduce multiple scattering, the disks for the HFBS sample cell had an annular shape with inner diameter of $20 \mathrm{~mm}$ and an outer diameter of $26 \mathrm{~mm}$ to match the beam width of $30 \mathrm{~mm}$. The circular disks for the DCS sample cell had no inner hole but a smaller 


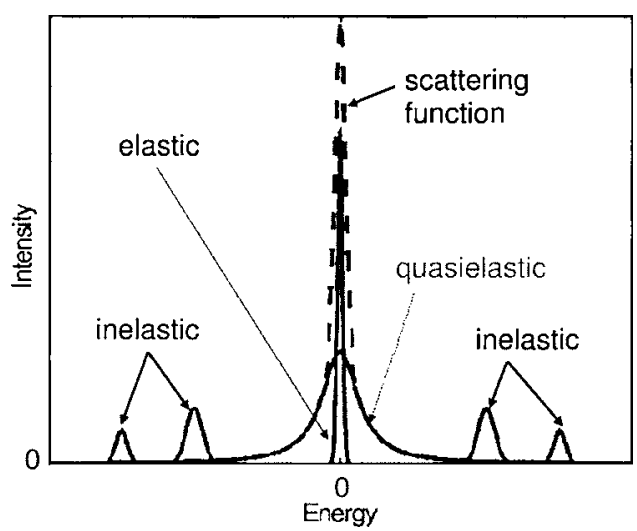

FIG. 2. A sketch showing qualitatively the elastic, the quasielastic, and inelastic parts of a spectrum. The abscissa is the energy transfer of the neutrons. It is positive when the neutrons have lost energy and negative when they have gained energy.

diameter of only $12.3 \mathrm{~mm}$. Both samples filled the full height of the beam, $30 \mathrm{~mm}$ (HFBS) and $100 \mathrm{~mm}$ (DCS), and the disks were stacked with their plane parallel to the scattering plane. They had a total mass and surface area, respectively, of $13.29 \mathrm{~g}$ and $222 \mathrm{~m}^{2}$ (HFBS) and $16.05 \mathrm{~g}$ and $356 \mathrm{~m}^{2}$ (DCS).

The spectra obtained by the two instruments contained features qualitatively similar to those sketched in Fig. 2. They consist of a peak centered around $\omega=0$, representing elastic scattering that is broadened relative to the instrumental resolution function by scattering from molecules in the monolayer undergoing diffusive motion (that is, dynamical modes of zero frequency). The features at larger $\omega$ in the DCS spectra reflect inelastic excitations in the sample such as vibrational and librational modes. The energy transfer is negative when the neutrons have gained energy from the sample and is positive for neutron energy loss.

In these studies, we focus on the quasielastic scattering around zero frequency and use the DAVE software ${ }^{19}$ to analyze the spectra from both spectrometers. The quasielastic spectra are fit to a scattering function given as the sum of a delta function at $\omega=0$ representing the elastic scattering, one or several Lorentzian functions representing the quasielastic scattering, and a linear background level function

$$
S_{s}(q, \omega)=A \delta(\omega)+\sum_{i} B_{i}\left(\frac{\Gamma_{i}}{\omega^{2}+\Gamma_{i}^{2}}\right)+I_{\text {backgr }}(\omega) .
$$

It must be folded with the instrumental resolution function $F_{\text {res }}$ to give a calculated intensity

$$
\operatorname{Intensity}(q, \omega)=\int_{-\infty}^{\infty} \mathrm{d} \omega^{\prime} S\left(q, \omega^{\prime}\right) F_{\text {res }}\left(q, \omega-\omega^{\prime}\right),
$$

where the parameters in Eq. (14) are determined by a fit to an observed spectrum. The resolution function is taken to be the spectrum at $100 \mathrm{~K}$, where it is assumed that all diffusive motion is frozen out and there is no broadening of the elastic peak.

On the DCS, we also observed vibrational and librational modes of the molecules in the inelastic spectra. This complicated the analysis of the molecular diffusive motions

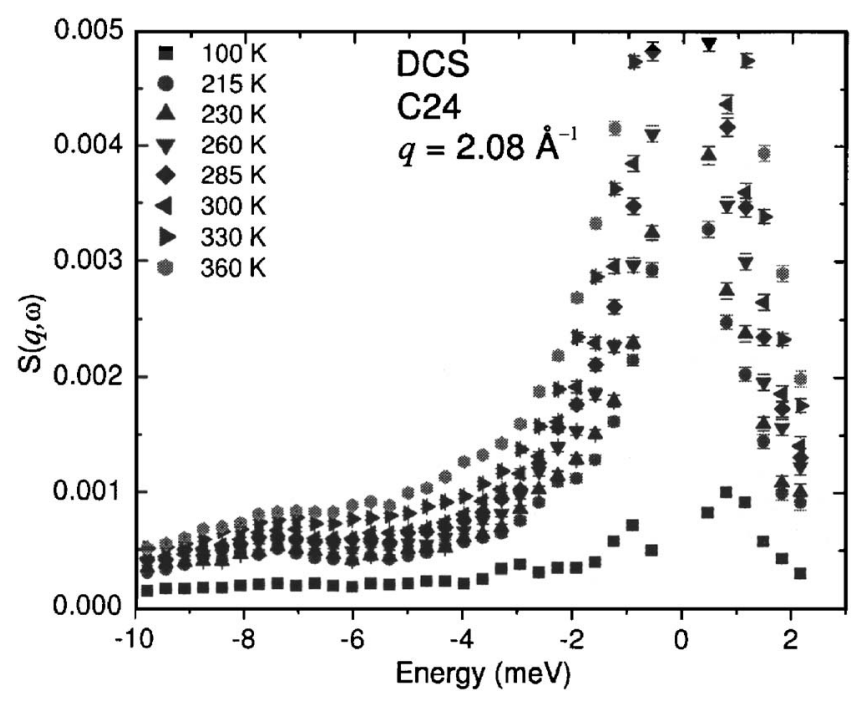

FIG. 3. Scattering functions measured on the DCS instrument from a 0.95layer $\mathrm{C} 24$ film at different temperatures at the wave vector transfer $Q$ $=2.08 \AA^{-1}$. For these spectra, the detectors have been divided into 41 groups. The incident neutron wavelength is $5.0 \AA$, corresponding to an instrumental energy resolution FWHM of $\sim 110 \mu \mathrm{eV}$. Error bars give the standard uncertainties ( \pm 1 standard deviation).

because these inelastic features overlapped with the quasielastic part of the spectra. Therefore, we needed a procedure for separating that part of a spectrum that could be used to fit the model in Eq. (14). We have devised a method, which will be described in the following section, for analyzing the quasielastic part of the DCS spectra that minimizes the influence of the inelastic part.

\section{A. Disk chopper spectrometer}

\section{Description of instrument and spectra}

The DCS is a "direct geometry" time-of-flight spectrometer. $^{13-15}$ A system of choppers, placed in the "white" beam from the reactor, is used to produce contaminant-free monochromatic pulses of neutrons at the sample. Scattering events are recorded in three banks of detectors placed a known distance from the sample. For each event, the scattered energy and therefore the energy transfer $\hbar \omega$ are determined, knowing the time of flight from sample to detector. Given the scattering angle, the wave vector transfer $q$ is also determined.

For the C24 (squalane) measurements the DCS was operated at an incident wavelength of $5.0 \AA$ (5.5 $\AA$ ), giving a full-width-at-half-maximum (FWHM) energy resolution of $\sim 110 \mu \mathrm{eV}(\sim 85 \mu \mathrm{eV})$ and a useful dynamic range of $\sim 10 \mathrm{meV}$, which allow probing processes on a time scale of 1-40 ps. The $q$ resolution of the instrument depends on how the 913 detectors are combined into groups equally spaced in $q$. There is a compromise to be made here. If one chooses a small number of groups, one has relatively good statistics but poor $q$ resolution, whereas with a large number of groups one has poorer statistics but better $q$ resolution. The grouping of detectors is specified in the figure captions of the spectra.

In Fig. 3 we present the scattering function $S_{s}(q, \omega)$ obtained on the DCS from a C24 monolayer at a series of temperatures from 100 to $360 \mathrm{~K}$. For comparison, in Fig. 4 


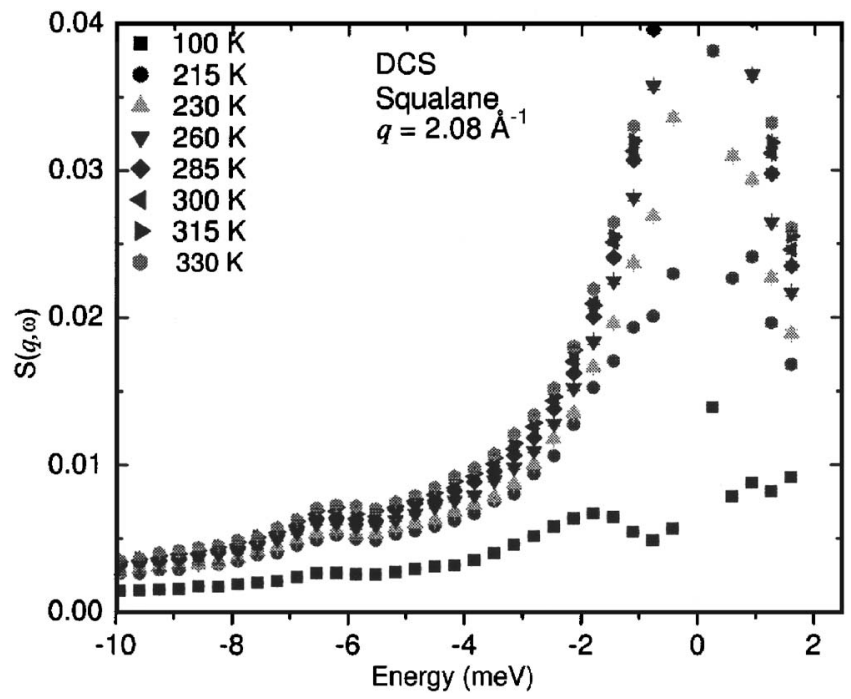

FIG. 4. Scattering functions measured on the DCS instrument from a 0.93 layer squalane film at different temperatures at the wave vector transfer $Q=2.08 \AA^{-1}$. For these spectra, the detectors have been divided into 46 groups. The incident neutron wavelength is $5.5 \AA$, corresponding to an instrumental energy resolution FWHM of $\sim 85 \mu \mathrm{eV}$. Error bars give the standard uncertainties $( \pm 1$ standard deviation).

we show the scattering function of a squalane monolayer obtained on the DCS at a series of temperatures from 100 to $330 \mathrm{~K}$. Both sets of spectra are presented at a wave vector transfer of $2.08 \AA^{-1}$ and show broad quasielastic peaks centered at a zero energy transfer, increasing in width as the temperature rises. Most of the data appear at a negative energy transfer $(\omega<0)$ since there is virtually no limit to the energy that a neutron can gain, whereas it cannot lose more than its initial energy (3.27 and $2.70 \mathrm{meV}$ for the C24 and squalane measurements, respectively). In both the C24 and squalane spectra, we clearly see evidence of vibrational and librational modes. In the $\mathrm{C} 24$ spectra, there is a peak between -7 and $-8 \mathrm{meV}$, and the spectrum at $100 \mathrm{~K}$ also contains peaks in the range of -1 to $-3 \mathrm{meV}$. In the squalane spectra, there is a peak between -6 and $-7 \mathrm{meV}$, while the spectrum at $100 \mathrm{~K}$ also contains a peak at $-2 \mathrm{meV}$. In addition, there seems to be a broad contribution to the inelastic spectra whose intensity increases with temperature in the $10 \mathrm{meV}$ neutron-energy-gain window. For both the C24 and squalane spectra, there is no clear boundary between the quasielastic and inelastic parts of the spectra, which makes it difficult to decide the appropriate energy range to use in the fit to the model function in Eq. (14).

\section{Data analysis}

We begin with an analysis of the squalane spectra in Fig. 4. At first, we arbitrarily chose the data in the range of -3 to $1.5 \mathrm{meV}$ for fitting to the scattering function in Eq. (14). We found that two Lorentzians were needed to give a reasonable fit to the data. The two Lorentzians had HWHM of about 1-2 meV and about $0.05 \mathrm{meV}$, depending on the temperature and $q$. This indicated that, in addition to a slow diffusive motion represented by the small width, there was a faster motion represented by the broad peak. Spectra calculated from the MD simulation data could only be fit to one
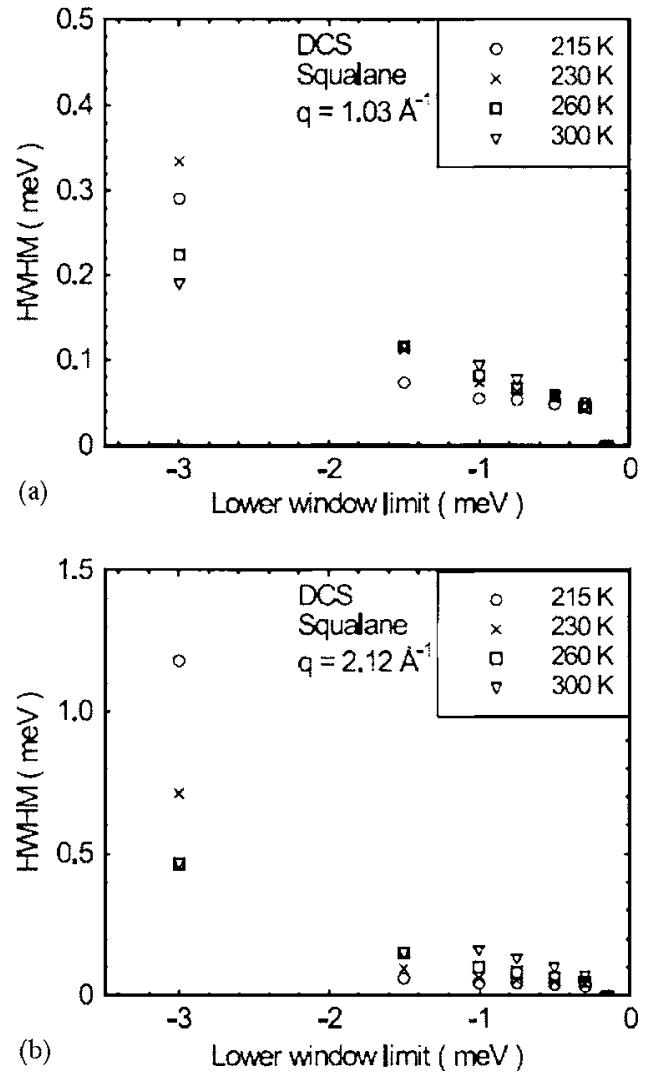

FIG. 5. The Lorentzian HWHM as a function of the low-energy limit of the energy interval about $\omega=0$ used in the fit of the observed DCS spectra for the squalane monolayer. Results are shown for two different wave vector transfers $q$ : (a) $1.03 \AA^{-1}$ and (b) $2.12 \AA^{-1}$. Error bars are smaller than the plotting symbols.

Lorentzian with a HWHM of about 20-40 $\mu \mathrm{eV}$, depending on $q$ and the temperature, so that it was impossible to identify a faster motion.

A closer look at the observed squalane spectrum in Fig. 4 at $100 \mathrm{~K}$ reveals that there is an inelastic peak at $\sim-2 \mathrm{meV}$ that was included in the fit. We identified this feature as the origin of the second Lorentzian with the large width. To reduce the contribution from the inelastic spectrum, we decreased the width of the energy window used and included only one Lorentzian in the fit. This had a great effect on the Lorentzian width, as shown in Fig. 5 for two different wave vector transfers $q$. Here, we have plotted the HWHM of the single Lorentzian as a function of the lower limit of the energy window used in the fit. When possible, the energy window is chosen symmetrically around the zero energy transfer. At both wave vector transfers, the drop in the HWHM is dramatic as we narrow the range of the fit around the zero energy transfer. This decrease is most pronounced at low temperatures. We see that the HWHM levels out and becomes almost independent of the energy window when the low-energy limit of the interval is chosen to be about $-1.5 \mathrm{meV}$ for the spectra taken at $q=2.12 \AA^{-1}$. If the energy range used for the fit is too narrow, it becomes impossible to fit the data. For this reason, we set the HWHM to zero in Fig. 5 when the low-energy limit of the interval is at $-0.15 \mathrm{meV}$

The choice of energy range for the fit should be such that 

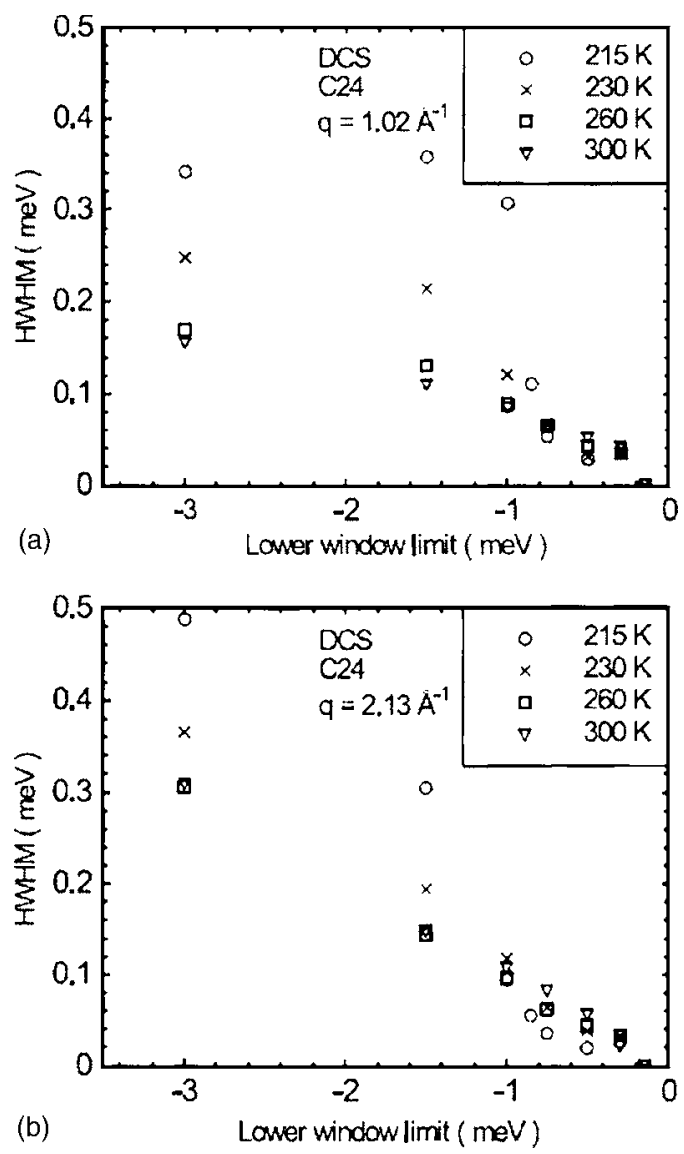

FIG. 6. The Lorentzian HWHM as a function of the low-energy limit of the energy range about $\omega=0$ used in the fit of the observed DCS spectra for the C24 monolayer. Results are shown at two different wave vector transfers $q$ : (a) $1.02 \AA^{-1}$ and (b) $2.13 \AA^{-1}$. Error bars are smaller than the plotting symbols.

the HWHM of the quasielastic scattering is nearly independent of the chosen interval. We note that at $q=2.12 \AA^{-1}$, the range in which the HWHM is insensitive to the energy range becomes smaller as the temperature increases. For the spectra at $q=1.03 \AA^{-1}$, we see that a constant value of the Lorentzian HWHM, independent of the energy interval, is not reached at the higher temperatures. It is therefore necessary to consider both the wave vector transfer and temperature before selecting the energy interval to be used in the fits.

The situation is not as optimal for the C24 spectra in Fig. 3, as is evident in Fig. 6. At both wave vector transfers, the Lorentzian HWHM in the low-temperature data nearly reaches a constant value in an interval beginning at $-0.75 \mathrm{meV}$; however, the high-temperature data for HWHM fail to reach a constant value.

In conclusion, it is very important to perform an analysis such as what we have done to determine the energy interval appropriate to fitting a Lorentzian to the quasielastic part of the spectra. Ideally, one should choose an interval such that the Lorentzian HWHM is independent of its width to insure that parts of the inelastic spectrum do not interfere with the fits. The choice of energy interval is complicated by a temperature dependence that causes the appropriate energy interval to decrease in width as the temperature increases. In the present work, we have chosen to do all the fits in the energy interval of -0.75 to $0.75 \mathrm{meV}$.

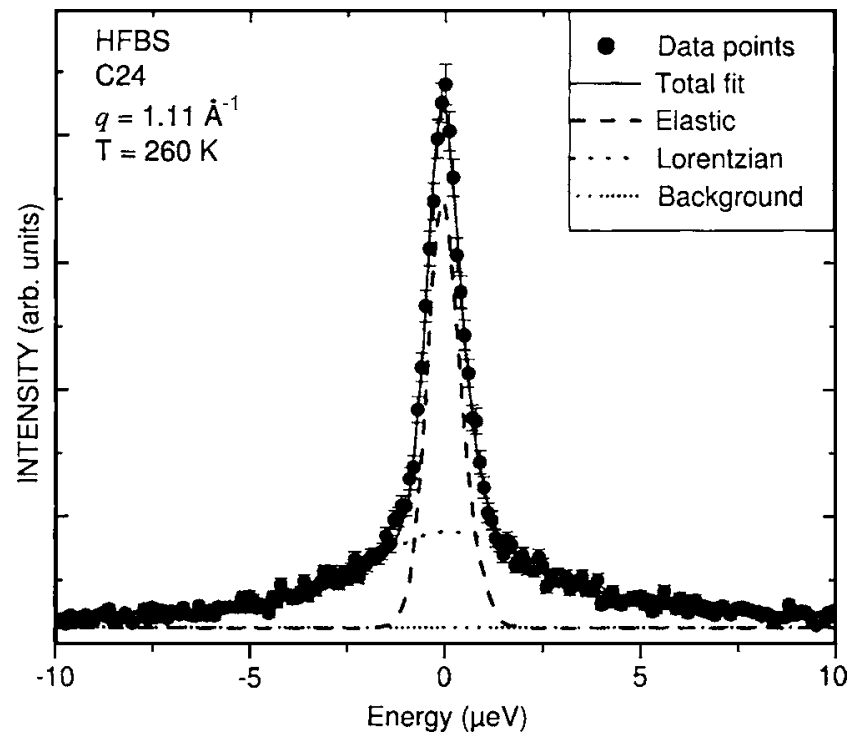

FIG. 7. Typical spectrum of a C24 monolayer measured on the HFBS instrument at wave vector transfer $Q=1.11 \AA^{-1}$. The figure illustrates the decomposition of the intensity into an elastic component (delta function) and a quasielastic (Lorentzian) component which are convoluted with the instrumental resolution function to give the long-dashed and short-dashed curves, respectively. The sample temperature is $260 \mathrm{~K}$. Error bars give the standard uncertainties ( \pm 1 standard deviation).

\section{B. High flux backscattering spectrometer}

In contrast to the DCS instrument, the HFBS instrument is configured in "inverse geometry": ${ }^{16,17}$ The incident energy is varied, while the scattered energy of the neutrons that reach the detectors is fixed. The white beam from the reactor is crudely monochromated using a helical velocity selector and then reflected by a phase space transformation (PST) chopper whose function is to increase the intensity at the sample by narrowing the wavelength distribution of neutrons that strike the monochromator. The monochromator crystals are mounted on a mechanical Doppler drive that periodically varies the incident energy of the neutrons within a narrow range, $36 \mu \mathrm{eV}$ on either side of the mean energy which is $2.08 \mathrm{meV}$. After scattering by the sample, neutrons with $2.08 \mathrm{meV}$ scattered energy are backscattered by the analyzer crystals and are counted in a set of detectors. Thus, the detected neutrons are those that have gained or lost energy in the sample corresponding to the Doppler-shifted incident energy. The PST chopper and the large solid angle subtended by the analyzer crystals greatly increase the counting rate of the HFBS, at the same time ensuring very good energy resolution, $\sim 1 \mu \mathrm{eV}$. The wave vector range of the instrument is from 0.62 to $1.75 \AA^{-1}$.

The HFBS spectrum from a C24 monolayer in Fig. 7 is used to illustrate the decomposition into a resolution-limited elastic component and the Lorentzian quasielastic component. The fitting parameters of interest are the Lorentzian HWHM $\left(\Gamma_{i}\right)$ in Eq. (14), the integrated intensity $\left(B_{i}\right)$ of the Lorentzian component, the elastic intensity $A$, and the background function $I_{\text {backgr }}(\omega)$.

\section{INELASTIC SPECTRA}

We investigated the types of molecular motions contributing to the DCS inelastic spectra in Figs. 3 and 4 in our MD 
simulations. For this purpose, we used the projection operator method developed by us ${ }^{11}$ to separate out the translational motion of the molecules along the $x$ and $y$ directions in the plane of the surface and along $z$, perpendicular to that plane. In the setup of the simulation, the long axis of the molecules was oriented along the $x$ axis. In addition, we also projected out the rotational motions of the molecules around the three principal axes of inertia. Fourier transformation of the calculated time correlation functions of the various motions yielded their power spectrum. Since some of the vibratory and libratory modes can be faster than the molecular diffusive motions, we used a shorter time step in these calculations compared to that given in Table I for the simulation of the quasielastic spectra. The time resolution in the simulations was set to $\tau=t_{\min }=0.03 \mathrm{ps}$ and the time window was set to $t_{\max }=5 \mathrm{ps}$, corresponding to a frequency resolution of $\Delta \nu=0.84 \mathrm{meV}$ and a dynamic range of $\nu_{\max }=69.1 \mathrm{meV}$.

\section{A. Tetracosane monolayer}

The frequency spectra at $300 \mathrm{~K}$ for the center-of-mass translational motion of the molecules and for the molecular rotational motion in the $\mathrm{C} 24$ monolayer are shown in Fig. 8. The power spectrum for the bouncing mode, $V_{z}$, in panel (a) has a broad peak at about $7.0 \mathrm{meV}$ close to the peak position observed for the C24 monolayer in Fig. 3. The spectra for the translational motions $V_{x}$ along $x$ and $V_{y}$ along $y$ are broader and rather featureless with the largest contributions appearing near zero frequency in the range of $0.8-2 \mathrm{meV}$. This behavior is also consistent with the experimental spectra in Fig. 3. We note that the power spectra of both the $x$ and $y$ motions have a finite value at zero frequency, which shows that these motions also have a diffusive character. Such lateral diffusion is consistent with the monolayer being in a smectic phase at $300 \mathrm{~K}$ as inferred by both the neutron diffraction experiments and MD simulations. ${ }^{1}$ In contrast, the bouncing mode is well separated from the quasielastic part of the spectrum and does not have a diffusive character.

The power spectrum for the rotational motion around the three principal axes of inertia is shown in Fig. 8(b) and covers the same frequency range as the translational motions. The spectrum for the rolling motion about the long axis of the molecule (Rot3) has a broad peak at $7 \mathrm{meV}$, the same frequency as that of the bouncing mode. The spectra for rotation around the other two principal axes are broad, extending from about $8 \mathrm{meV}$ down to zero frequency with a considerable diffusive component as well.

\section{B. Squalane monolayer}

The power spectra calculated for translational and rotational motions in a squalane monolayer at $215 \mathrm{~K}$ are shown in Fig. 9. The inelastic spectra for the squalane and C24 monolayers are quite similar. The bouncing mode of the squalane monolayer is shifted slightly to a lower frequency compared to that of $\mathrm{C} 24$, a shift that is also observed in the experimental spectra in Fig. 4. The shift reflects a slight change in the ratio between the molecule-substrate force constant and the molecular mass. Squalane is heavier; in addition, there are other contributions to the force constant from
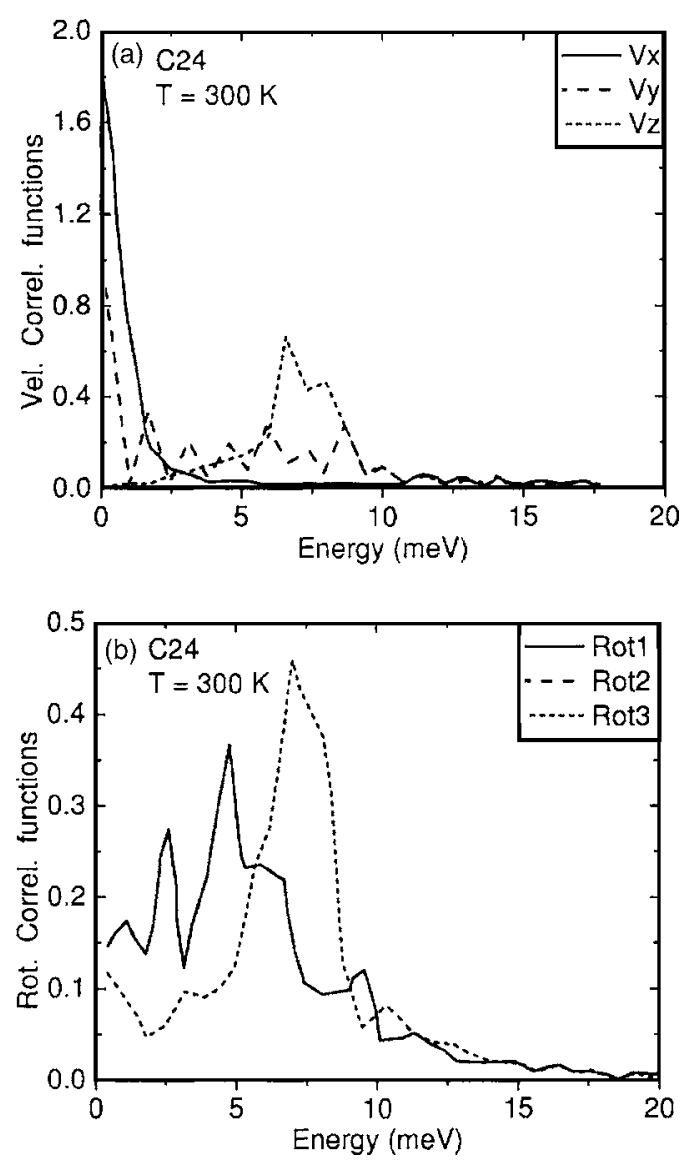

FIG. 8. (a) The time Fourier transform of the velocity correlation function (the power spectrum) for the three Cartesian components $V_{x}, V_{y}$, and $V_{z}$ of the center-of-mass velocity of the molecules in a C24 monolayer. (b) The power spectra of the rotational velocity correlation functions for rotations Rot1, Rot2, and Rot3 about the three principal axes of inertia (in order of descending moments of inertia). Rot 3 is a rotation about the long axis of the C24 molecule, the axis with the smallest moment of inertia. Note that the results for rotations around Rot 1 and Rot 2 are practically degenerate so that only two curves are seen in the plot (b). The temperature is $300 \mathrm{~K}$. No quantitative uncertainty analysis was performed, but the calculations are based on MD simulation results with a standard deviation of the total energy on the $1 \%$ level.

the methyl side groups. There also seems to be a peak in the velocity correlation functions for lateral motion at about $2 \mathrm{meV}$, as observed in the experimental spectra. The contribution from the rolling motion about the long axis of the molecule (Rot3) has a peak at the same frequency as the bouncing mode, whereas the contributions from the other rotational motions to the spectrum are broad and span the region from zero to $20 \mathrm{meV}$, just as for $\mathrm{C} 24$. When we look at the power spectra near zero frequency, we see a difference between the C24 and squalane monolayers. There is a clearly less diffusive motion in the squalane monolayer at $215 \mathrm{~K}$ than in the $\mathrm{C} 24$ monolayer at $300 \mathrm{~K}$ since the diffusive motion becomes more pronounced at higher temperatures.

In summary, the observed inelastic spectra of the two monolayers are qualitatively similar. They both show intensity that grows with temperature over a wide energy interval. The power spectra show that this contribution reflects lateral translational motion as well as rotational motions of the molecules, primarily the Rot 1 and Rot 2 contributions. The peak at -7 to $-8 \mathrm{meV}$ is caused by the bouncing mode and by the 

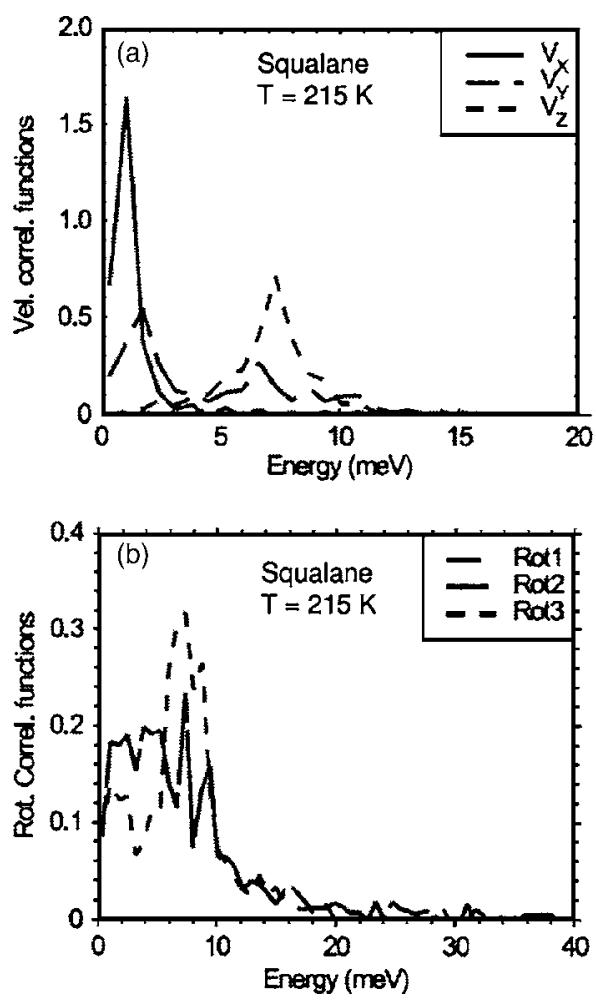

FIG. 9. (a) The time Fourier transform of the velocity correlation function (the power spectrum) for the three Cartesian components $V_{x}, V_{y}$, and $V_{z}$ of the center-of-mass velocity of the molecules in a squalane monolayer. (b) The power spectrum of the rotational velocity correlation function for rotations Rot1, Rot2, and Rot3 about the three principal axes of inertia (in order of descending moments of inertia). Rot 3 is a rotation about the long axis of the squalane molecule, the axis with the smallest moment of inertia. Note that the rotational motions about Rot 1 and Rot 2 are practically degenerate, so only two curves are seen in the plot (b). The temperature is $215 \mathrm{~K}$. No quantitative uncertainty analysis was performed, but the calculations are based on MD simulation results with a standard deviation of the total energy on the $1 \%$ level.

uniaxial rotation about the long axis of the molecule, Rot3. The peak at $-2 \mathrm{meV}$ in the squalane monolayer can be explained primarily as a lateral translational motion. Only the bouncing modes are well separated from the quasielastic part of the spectra, whereas the other modes have low-frequency components that merge with the quasielastic spectra.

\section{QUASIELASTIC SPECTRA}

Quasielastic spectra provide information about the diffusive, or zero frequency, modes in a system. By conducting measurements at a wave vector transfer $q$, we obtain information about the diffusive motion on a length scale of $\sim 1 / q$. We have already described the difficulty of separating the quasielastic and inelastic spectra. This will always be the case for monolayers of complex molecules that have a broad inelastic power spectrum extending to very small frequencies. In the following, we will describe and discuss the quasielastic scattering spectra of the C24 and squalane monolayers obtained on the DCS and HFBS instruments, and we will compare them with MD simulations of the spectra. We use the projection operator method ${ }^{11}$ in the analysis of the quasielastic spectra, as we did in the analysis of the inelastic spectra.

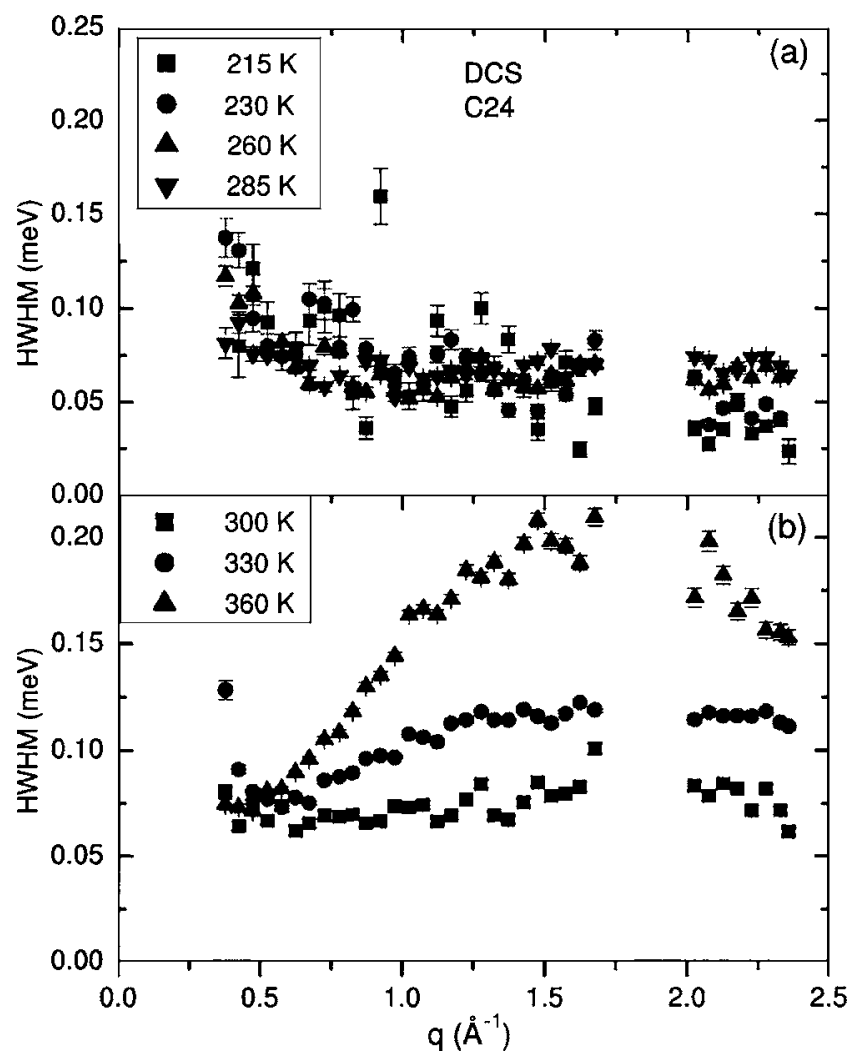

FIG. 10. The HWHM of the Lorentzian component of the quasielastic scattering from a C24 monolayer as measured on the DCS instrument: (a) below room temperature and (b) above room temperature. The fits to the spectra have been confined to the energy range of -0.75 to $+0.75 \mathrm{meV}$. Data are not shown in the $q$ range from 1.7 to $2.0 \AA^{-1}$ because of interference by a Bragg peak from the graphite substrate. Error bars give the standard uncertainties ( \pm 1 standard deviation).

\section{A. DCS quasielastic spectra}

\section{Tetracosane monolayer}

The $q$ dependence of the width of the quasielastic scattering from the $\mathrm{C} 24$ monolayer as measured on the DCS is shown in Fig. 10 at a series of temperatures. The HWHM is essentially dispersionless ( $q$ independent) except at the highest temperatures at 330 and $360 \mathrm{~K}$, where we see an increase in the HWHM on heating. The dispersionless width may indicate that the quasielastic scattering is caused by rotational diffusive motion of the molecules in the monolayer, as we have discussed earlier. The origin of the increase in the HWHM at higher temperatures is not clear. One possibility is translational diffusive motion, since that would produce a $q$-dependent HWHM, as we have discussed in Sec. II B. We have seen from the elastic diffraction measurements ${ }^{20}$ that, above $215 \mathrm{~K}$, the $\mathrm{C} 24$ monolayer is in a smecticlike phase that melts at $340 \mathrm{~K}$. We would expect that the translational diffusive motion in the smectic phase corresponds to a slow process as depicted in Fig. 1 and that the rate of the diffusional motion would increase with temperature. The intensity from such a slow diffusive motion would then end up in the elastic peak as sketched in Fig. 1(c). This behavior and also the origin of the drop in the HWHM as $q$ increases $\left(q<0.75 \AA^{-1}\right)$ in the temperature range $215-285 \mathrm{~K}$ have been investigated in the MD simulations.

The MD simulations showed that it was only possible to 


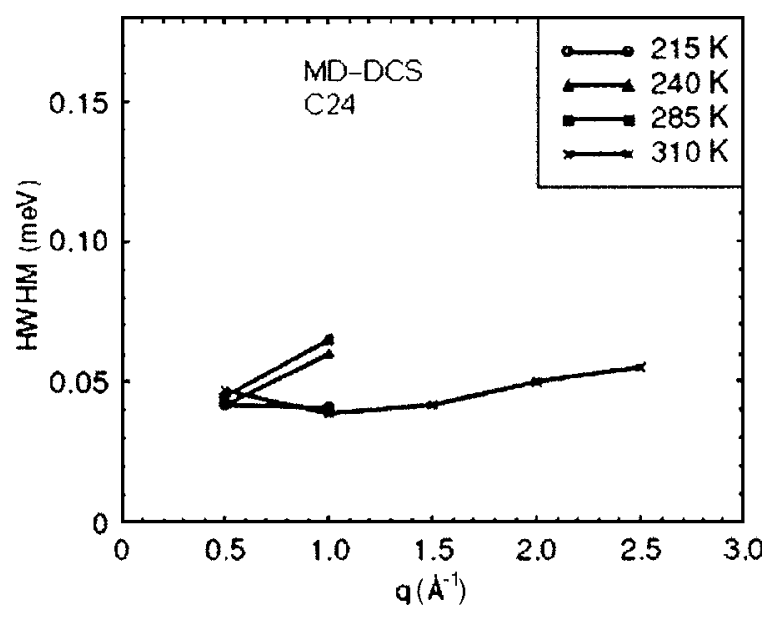

FIG. 11. The Lorentzian HWHM of the scattering function contributed by rotational and intramolecular diffusive motions calculated from MD simulations of a C24 monolayer. The HWHM is plotted vs $q$ for various temperatures, and the scattering function is calculated for a time resolution and dynamic range corresponding to the DCS. No quantitative uncertainty analysis was performed, but the calculated data points are based on MD simulation results with a standard deviation of the total energy on the $1 \%$ level.

monitor the translational diffusive motion at wave vectors up to $0.5-1.0 \AA^{-1}$. At higher $q$, the translational diffusion became too fast relative to the dynamic range of the DCS. The simulations showed that the intensity of the quasielastic scattering contributed by molecules undergoing translational diffusion became weaker and ended up as a uniform contribution to the background scattering, as in the case of the fast mode in Fig. 1(c), so that quasielastic scattering from rotational and intramolecular motions dominates the spectra. We calculated the HWHM of the quasielastic scattering produced by these motions using the projection technique described in Ref. 11. As shown in Fig. 11, we were only able to determine a HWHM for all $q$ at the highest temperature and for $q<1 \AA^{-1}$ at the lower temperatures. The reason is that we are just at the limit of the diffusive motion being too fast for the time resolution chosen in the simulations. This causes a lot of numerical noise in the calculation of the spectra and makes it impossible to determine a reliable HWHM. It may seem counterintuitive that the diffusive processes become slower at higher temperature where we could determine a HWHM. However, there are conformational changes of the molecules that slow down the rotational diffusion. ${ }^{4}$ At $310 \mathrm{~K}$, gauche defects are formed along the molecular chains, making the molecules more globular in shape. ${ }^{21}$ The principal moments of inertia then increase, causing the rotational diffusive motion to be slower.

It follows from this discussion that the increase observed in the Lorentzian HWHM at the high temperatures in Fig. 10 (b) cannot reflect a translational diffusive motion; its rate increases with temperature, and it is already too fast at low temperature to be within the dynamic range of DCS. A closer look at the data reveals that the increase in HWHM is more likely to be related to the data fitting procedure. Recall that the spectra were fitted in the energy interval $(-0.75,0.75) \mathrm{meV}$, which is appropriate at low temperatures. However, we see from Fig. 6(a) that the fit should be done in

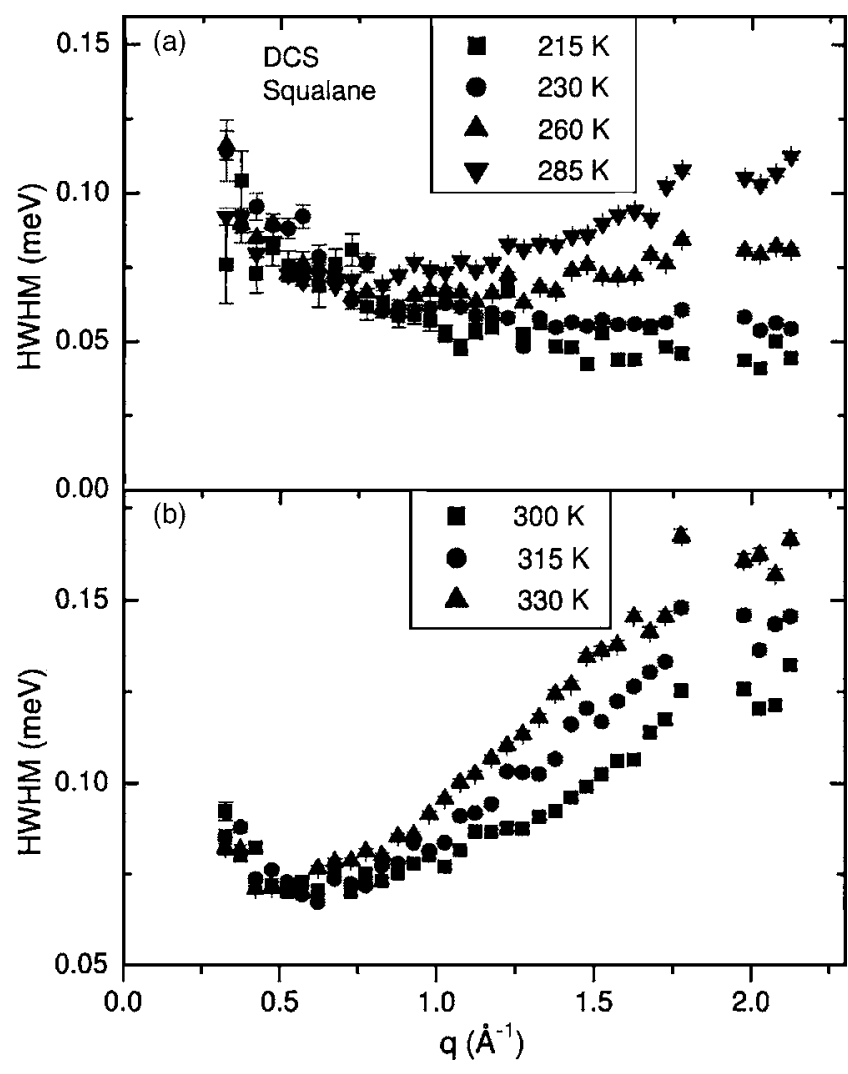

FIG. 12. The HWHM vs $q$ of the Lorentzian component of the quasielastic scattering from a squalane monolayer as measured on the DCS instrument: (a) below room temperature and (b) above room temperature. The fits to the spectra have been confined to the energy range of -0.75 to $+0.75 \mathrm{meV}$. Data are not shown in the $q$ range from 1.7 to $2.0 \AA^{-1}$ because of interference by a Bragg peak from the graphite substrate. Error bars give the standard uncertainties $( \pm 1$ standard deviation)

a narrower energy interval at higher temperatures because the HWHM already depends strongly on the width of the interval at a lower limit of $-0.75 \mathrm{meV}$. The data should instead have been fitted over an interval of, say, $(-0.4,0.4) \mathrm{meV}$ where Fig. 6(a) shows that the observed HWHM at $300 \mathrm{~K}$ is essentially equal to that at lower temperatures and close to the value given by simulations in Fig. 11. Thus, it appears likely that the increase in the HWHM above $300 \mathrm{~K}$ in Fig. 10(b) is an artifact of the fitting procedure and not a real effect due to the overlap of the broad band of inelastic scattering with quasielastic scattering.

The MD simulations also suggest an explanation for the drop in HWHM as $q$ increases at small $q$ at the lower temperatures in Fig. 10(a). The simulations showed that up to about $q \sim 1 \AA^{-1}$ the translational diffusive motions contribute to the quasielastic scattering, but at higher $q$ the motions are too fast to lie within the dynamic range of the DCS so that the intensity decreases into the smooth background scattering. That is, the decrease in HWHM at low $q$ may be caused by the contribution from translational diffusive motions gradually disappearing from the quasielastic spectra and ending up in the background, while the slower rotational and intramolecular diffusive motions are still within the DCS' dynamic range. There is no drop in HWHM as $q$ increases at the higher temperatures in Fig. 10(b) because the translational diffusive motions are already too fast at even the lowest $q$. 


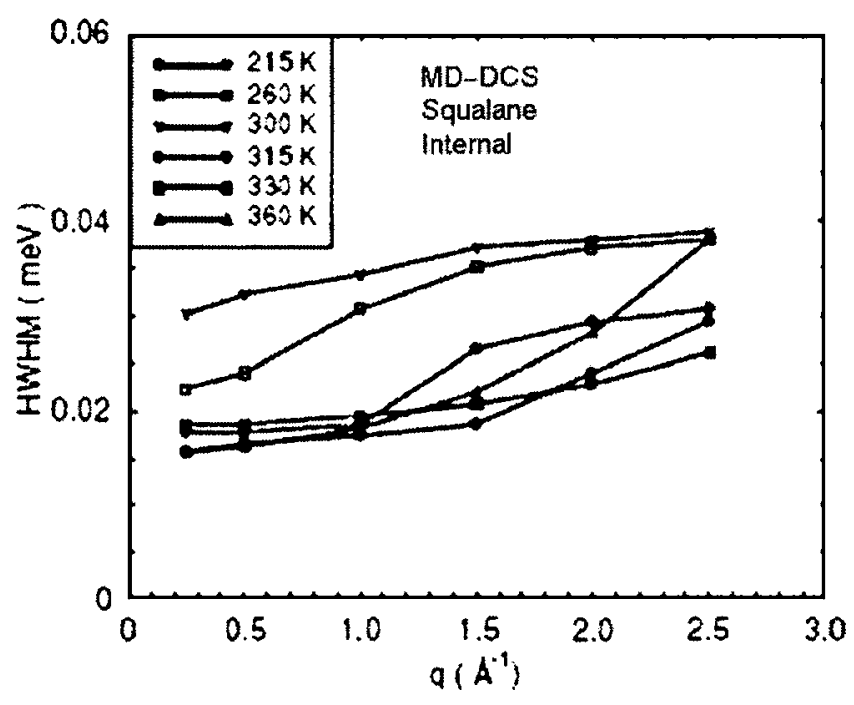

FIG. 13. MD simulation of the squalane monolayer scattering function HWHM corresponding to measurements on the DCS. The Lorentzian HWHM is plotted vs wave vector transfer $q$. Only contributions from rotational and intramolecular motions are included. No quantitative uncertainty analysis was performed, but the calculated data points are based on MD simulation results with a standard deviation of the total energy on the $1 \%$ level.

\section{Squalane monolayer}

The $q$ dependence of the Lorentzian HWHM for the squalane monolayer is shown in Fig. 12 at a series of temperatures as measured on the DCS. We have seen from the elastic diffraction measurements ${ }^{1}$ that there is a transition from a crystalline to a smectic phase at $215 \mathrm{~K}$, followed by a melting transition at $325 \mathrm{~K}$. Similar to the C24 monolayer, we see in Fig. 12(a) a slight drop in the HWHM at low temperature as $q$ increases up to about $1 \AA^{-1}$ and then a slight increase in the HWHM with temperature. In contrast to C24, we also see in Fig. 12(b) a slight drop in the HWHM as $q$ increases at high temperatures for $q$ values up to $\sim 0.5 \AA^{-1}$ and, at larger $q$, a stronger increase in the HWHM with $q$ than for the $\mathrm{C} 24$ monolayer.

The MD simulation results are shown in Fig. 13. As in the case of the $\mathrm{C} 24$ monolayer, we found that the translational diffusive motion was too fast to be detected for $q$ $>0.5 \AA^{-1}$, so it has been excluded from the simulations by the projection operator technique. The HWHM therefore represents the contributions from the rotational and intramolecular diffusive motions. We have further decomposed the HWHM in Fig. 13 into rotational and intramolecular contributions, as shown in Fig. 14. The results reveal an interesting detail in the dynamics. We see in Fig. 14(a) that the HWHM of the rotational diffusive motion increases with temperature up to $300 \mathrm{~K}$ and then drops to a level slightly below that at $215 \mathrm{~K}$ at higher temperatures. Also, the intramolecular motions were too fast to be detected in the quasielastic spectra for temperatures below $300 \mathrm{~K}$, but in Fig. 14(b), we see that they become slower above $300 \mathrm{~K}$ so they could be detected with the DCS instrument. This behavior is consistent with structural changes that take place in the squalane monolayer. As discussed in Ref. 1, the number of gauche defects along the chains increases at $300 \mathrm{~K}$, making the molecule more globular in shape. The resulting increase in the moments of
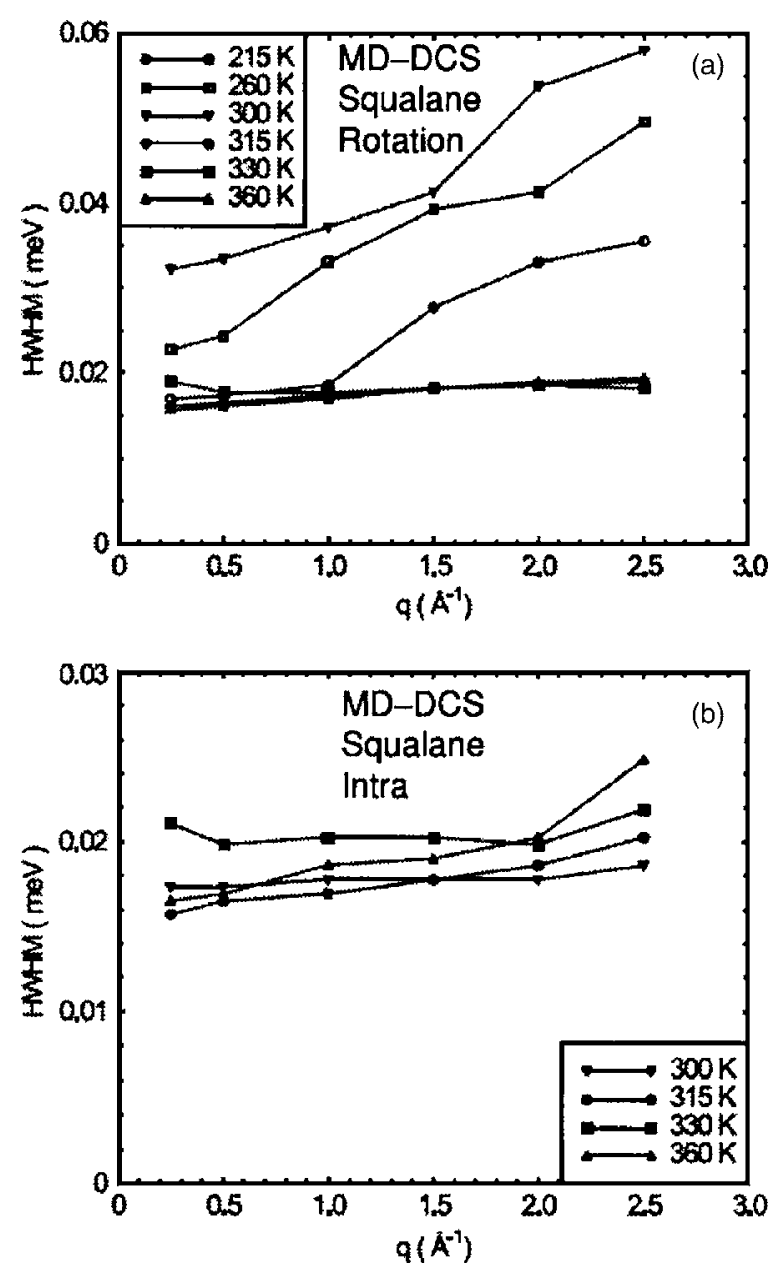

FIG. 14. The HWHM of the quasielastic scattering from (a) rotational and (b) intramolecular motions obtained from MD simulations of the DCS spectra. The HWHM vs $q$ is plotted for various temperatures. No quantitative uncertainty analysis was performed, but the calculated data points are based on MD simulation results with a standard deviation of the total energy on the $1 \%$ level.

inertia causes a decrease in the rate of rotational diffusion and hence a drop in the HWHM for the rotational motions. Below $300 \mathrm{~K}$, the intramolecular motions contribute to the inelastic spectra mainly as librational motions; however, at higher temperatures as seen in Fig. 14(b), the motions associated with the creation and annihilation of the gauche defects contribute to the quasielastic spectra on a time scale accessible to the DCS. In the next section, we will see that this intramolecular motion also contributes to the HFBS spectra.

\section{B. HFBS quasielastic spectra}

\section{Tetracosane monolayer}

The HWHM of the Lorentzian component in the quasielastic spectra of the C24 monolayer measured on the HFBS is shown in Fig. 15 plotted versus $q$ at several temperatures. ${ }^{4}$ The low-temperature data have been taken with a dynamic range of $\pm 11 \mu \mathrm{eV}$, and the high-temperature data where the diffusive motion is faster have been taken with a dynamic range of $\pm 35 \mu \mathrm{eV}$.

At 215 and $230 \mathrm{~K}$, the HWHM is dispersionless, consistent with the uniaxial rotation of the molecules about their 


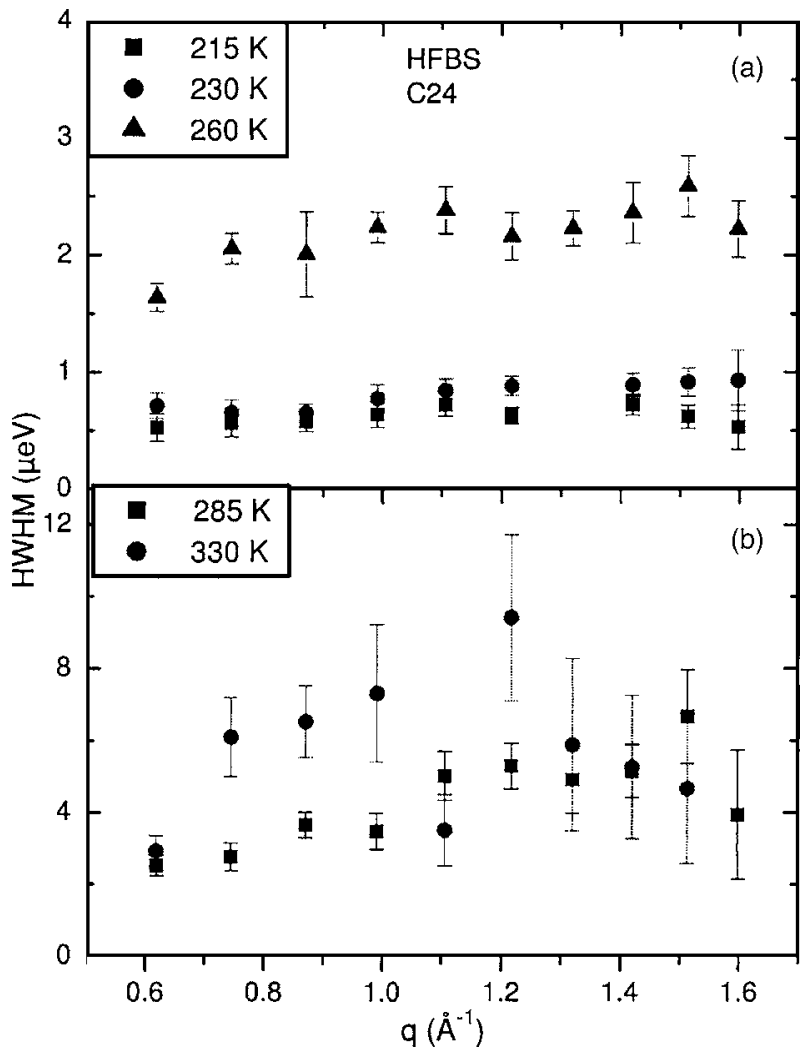

FIG. 15. The HWHM of the Lorentzian component of the quasielastic scattering of the $\mathrm{C} 24$ film of thickness of 0.94 layers (Ref. 4) as measured on the HFBS instrument. The dynamic range is $\pm 11 \mu \mathrm{eV}$ in (a) and $\pm 35 \mu \mathrm{eV}$ in (b). Error bars give the standard uncertainties $( \pm 1$ standard deviation).

long axis. Above $230 \mathrm{~K}$, the width begins to increase with temperature, implying a faster motion; we also see a slight increase in the width with $q$. This indicates that the motion may no longer be characterized as a simple uniaxial rotation and that it is probably coupled to other types of motion in the system. Our MD simulations at a series of temperatures indicate that the diffusive translational motion is too fast for the time resolution in the HFBS simulations. In the following, we will focus on the results at three temperatures, 180, 260, and $330 \mathrm{~K}$, as shown in Figs. 16 and 17.

The low-temperature simulations at $180 \mathrm{~K}$ showed that the translational diffusive motion was too fast for the time resolution used in the simulations. The time resolution was adequate for monitoring the rotational diffusive motion at wave vector transfers up to $q=1.5 \AA^{-1}$, beyond which the motions again became too fast so that, as discussed in Sec. II A, the quasielastic intensity ends up in the background. We analyzed the rotational contribution to the quasielastic scattering in some detail in order to determine its character, anticipating that at $180 \mathrm{~K}$ we would have a rather "clean" example of a uniaxial rotation about the long molecular axis. To test this idea, we compared the simulation results with that calculated in Eq. (11) for a uniaxial rotation. We used both the elastic intensity, as given by the nonzero level to which the intermediate scattering function decays [see the sketch in Fig. 1(b)], and the intensity of the quasielastic peak. These were fitted to the theoretical expression in Eq. (11) with $m=1$ for the quasielastic contribution in order to

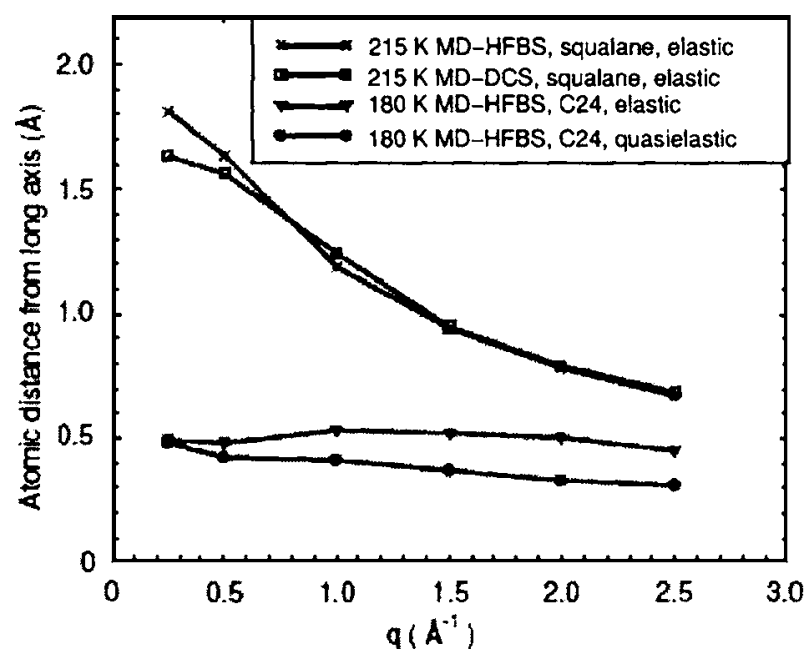

FIG. 16. The average atomic distance $r$ from the long axis of the molecules determined by fitting the simulated HFBS spectra to a model of uniaxial rotation about this axis. For the $\mathrm{C} 24$ molecule, we used both the elastic and quasielastic parts of the simulated HFBS scattering at $180 \mathrm{~K}$. For squalane, we used the elastic scattering from both the simulated DCS and HFBS spectra at $215 \mathrm{~K}$. No quantitative uncertainty analysis was performed, but the calculated data points are based on MD simulation results with a standard deviation of the total energy on the $1 \%$ level.

determine $r$, the distance of the atoms from the axis of rotation. The theoretical value of $r$ for the pseudoatom molecule in the all-trans configuration is $0.44 \AA$. The results are shown in Fig. 16. Ideally, the $r$ value should be independent

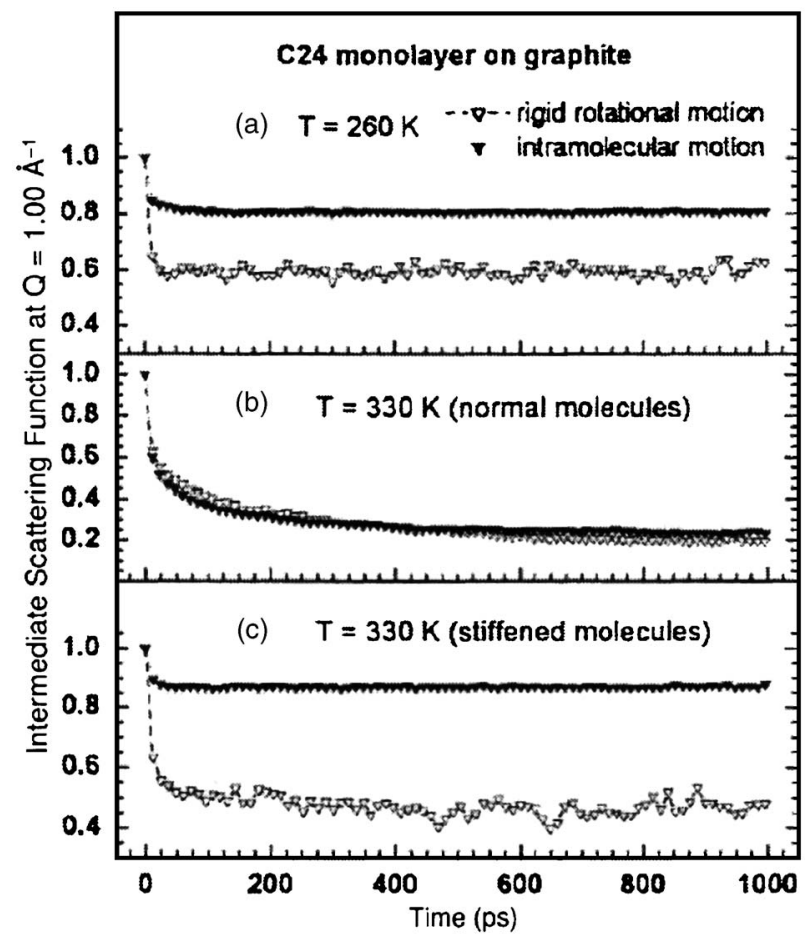

FIG. 17. MD simulation of the intermediate scattering function $F_{s}(q, t)$ for rotational and intramolecular motion of a $\mathrm{C} 24$ monolayer. In (c) the torsion potential was increased to stiffen the molecule. A comparison of (b) and (c) shows that the reappearance of the rotational motion in (b) is due to the introduction of gauche defects in the flexible C24 molecules (from Ref. 4). No quantitative uncertainty analysis was performed, but the calculated data points are based on MD simulation results with a standard deviation of the total energy on the $1 \%$ level. 
of $q$, and this is almost the case with a value close to the theoretical value. So, we may conclude that the quasielastic scattering observed at low temperatures on the HFBS is due to the uniaxial rotation about the long axis of the molecule. A similar conclusion was reached for a C32 monolayer. ${ }^{12}$

At $260 \mathrm{~K}$, we found, as at $180 \mathrm{~K}$, that the translational motion made no contribution to the quasielastic scattering. In addition, the rotational diffusive motion became too fast to lie within the dynamical range of the HFBS. This is illustrated in Fig. 17 by the intermediate scattering function calculated from the simulations for the rotational and intramolecular motions at $q=1 \AA^{-1}$ and $260 \mathrm{~K}$. The intermediate scattering functions for these motions look just like those for the fast modes sketched in Fig. 1. This is in contrast to the HFBS experiments that gave some quasielastic scattering at this temperature, as seen in Fig. 15. There are two reasons for this. One is the difference in energy resolution in the experiments and simulations as seen in Table I. The simulations were done with an energy resolution of $1.7 \mu \mathrm{eV}$ to reduce the computation time compared to the $\sim 1.0 \mu \mathrm{eV}$ resolution in the experiments. We note that the experimental HWHM at $260 \mathrm{~K}$ is just about the energy resolution in the simulations. Another reason for this discrepancy between the HFBS experiments and the simulations is a difference in the moments of inertia between those of the real molecule and those of the pseudoatom molecule used in the simulations. With the molecule in the all-trans conformation, the moment of inertia for rotation about the long axis of the molecules is about a factor of 2 smaller in the pseudoatom model of the molecule than in the real molecule. This leads to a rotational diffusion constant that may be about a factor of $\sqrt{2}$ larger in the model. Thus, the quasielastic intensity for the dominant uniaxial rotation would end up in the smooth background in the simulations.

It is interesting to see in Fig. 17 that the intermediate scattering function at $330 \mathrm{~K}$ has a slower decay, suggesting that slower rotational and intramolecular motions have been activated. The temperature of $330 \mathrm{~K}$ is just $10 \mathrm{~K}$ below the C24 monolayer melting temperature, where the MD simulations indicate that many molecules have gauche defects both in the central region of the chains as well as at the chain ends. We have shown that the appearance of quasielastic scattering from the intramolecular motions and the reappearance of quasielastic scattering from rotational diffusion reflect the creation and annihilation of gauche defects. ${ }^{4}$ These defects make the molecules more globular in shape, thereby increasing the moments of inertia and reducing the rate of rotational diffusion. The scattering from the rotational diffusive motion thus occurs in the dynamical range of the HFBS. At the same time, there will also be some slow intramolecular diffusive motions associated with the creation and annihilation of gauche defects along the chain. In Fig. 17(c), we have shown the intermediate scattering function calculated for stiffened molecules so that no gauche defects are formed. Then we again see an intermediate scattering function characteristic of a fast process, supporting the interpretation of the dynamical slowdown evident in Fig. 17(b) as being caused by gauche defects.

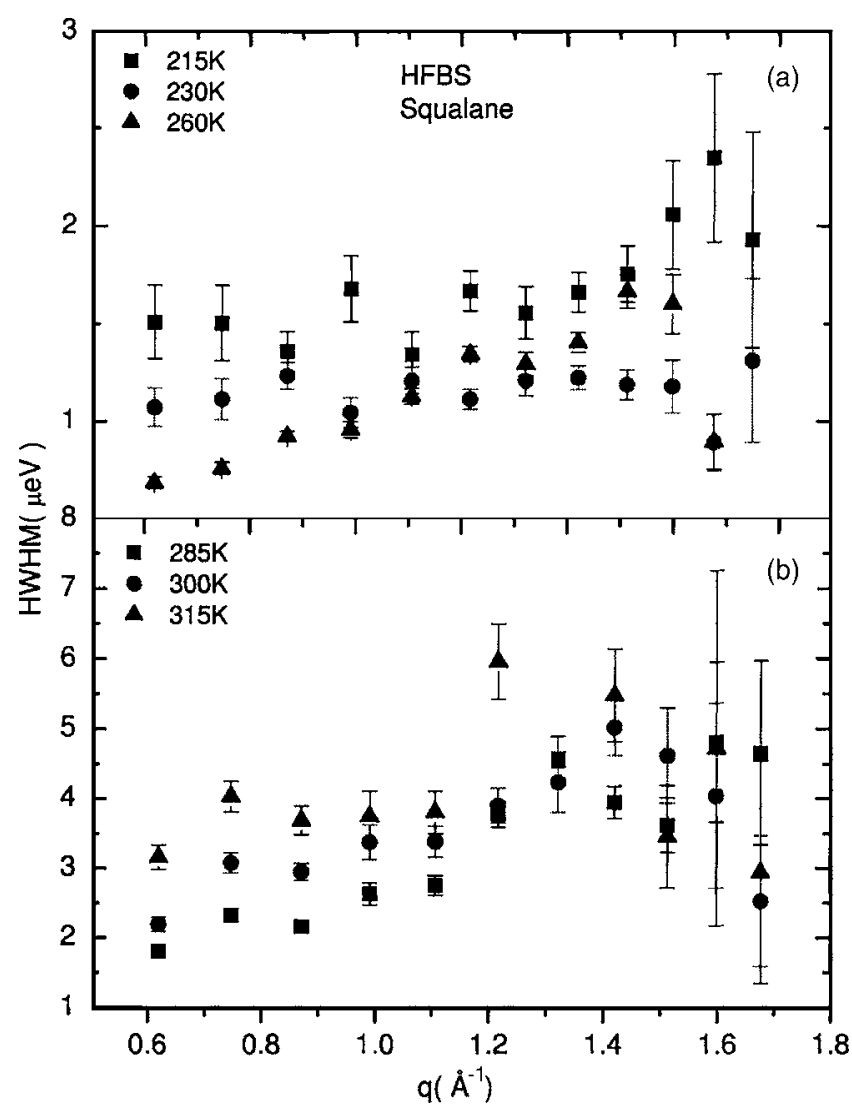

FIG. 18. The HWHM of the Lorentzian component of the quasielastic scattering from a squalane film of thickness of 0.95 layers as observed on the HFBS. The dynamic range is $\pm 11 \mu \mathrm{eV}$ in (a) and $\pm 35 \mu \mathrm{eV}$ in (b). Error bars give the standard uncertainties $( \pm 1$ standard deviation).

\section{Squalane monolayer}

Figure 18 shows the $q$ dependence of the Lorentzian HWHM obtained by fitting the HFBS quasielastic spectra from a squalane monolayer at a series of temperatures. The measurements were carried out using two different dynamical ranges as for the $\mathrm{C} 24$ monolayer. We note that for $q<1.2 \AA^{-1}$ the Lorentzian width decreases with increasing temperature up to $260 \mathrm{~K}$ and that the width at $260 \mathrm{~K}$ crosses that for $230 \mathrm{~K}$ at $q=1.2 \AA^{-1}$. The decrease in HWHM with increasing temperature is somewhat surprising, and we offer a possible explanation from the MD simulations as described below. At temperatures above $285 \mathrm{~K}$ the width increases monotonically with temperature as expected.

In the MD simulations of the squalane monolayer, the translational diffusive motion is too fast to contribute to the quasielastic scattering. In contrast to the $\mathrm{C} 24$ monolayer, the rotational and intramolecular motions are slow enough to be monitored in the simulations due to a larger moment of inertia about the long axis of the molecule. The HWHM of the simulated quasielastic scattering is shown in Fig. 19. It is almost dispersionless as found experimentally, and, as expected, the main contributions are from rotational motions. The spectra have been decomposed into a rotational and an intramolecular contribution with their respective HWHM as shown in Fig. 20. As in the experiments, we find a small decrease in the width of the rotational contribution [Fig. 20(a)] for $q<1.5 \AA^{-1}$ as the temperature increases in the 


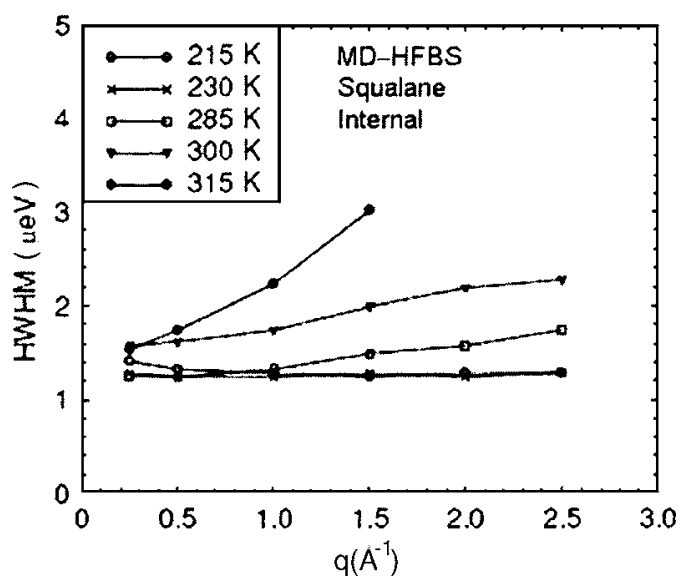

FIG. 19. MD simulation of the Lorentzian HWHM of the quasielastic scattering for comparison with the HFBS spectra of the squalane monolayer. There are only contributions from rotational and intramolecular motions. No quantitative uncertainty analysis was performed, but the calculated data points are based on MD simulation results with a standard deviation of the total energy on the $1 \%$ level.

range of $215-285 \mathrm{~K}$, above which the HWHM increases with temperature for all $q$. We know from simulations of the monolayer structure that in this temperature range there are conformational changes in the molecules from right-to-left gauche forms associated with the methyl side groups. ${ }^{1}$ These conformational changes may be responsible for this peculiar $q$-dependent behavior of the HWHM. In addition, we note from Fig. 20(b) that contributions from the intramolecular
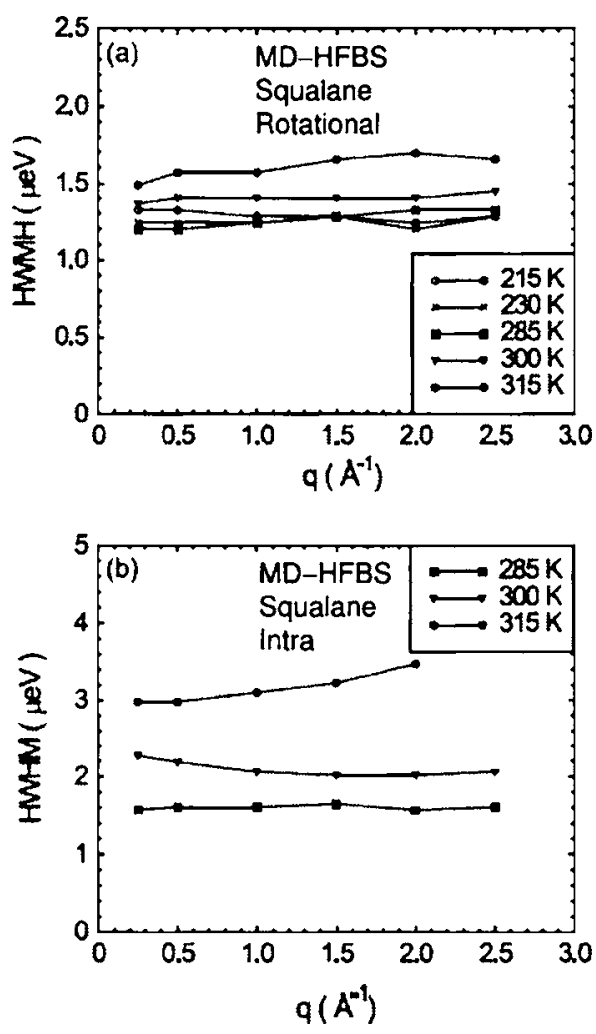

FIG. 20. Lorentzian HWHM of (a) the rotational and (b) the intramolecular contributions to the simulated HFBS spectra for the squalane monolayer. No quantitative uncertainty analysis was performed, but the calculated data points are based on MD simulation results with a standard deviation of the total energy on the $1 \%$ level. motions to the quasielastic scattering appear at a lower temperature $(285 \mathrm{~K})$ in the squalane monolayer than in the C24 monolayer, where they are first detected at $330 \mathrm{~K}$. Their appearance at low temperature is also due to excitations associated with the side groups in the squalane molecule.

As in the case of the $\mathrm{C} 24$ monolayer, we have used the low-temperature simulations at $215 \mathrm{~K}$ to determine the average distance $r$ of the atoms from the long axis of the molecule, anticipating that the low-temperature scattering is dominated by a uniaxial rotation about this axis. The results shown in Fig. 16 are based on the elastic part of the scattering calculated from the simulated spectra for both the DCS and HFBS. Since $r$ has a strong dependence on $q$, we may conclude that the motion is more complicated than that given by a simple uniaxial rotation. Compared to the $\mathrm{C} 24$ monolayer results, we see that $r$ is larger as expected. The structural distortion we have seen in the squalane molecules upon adsorption ${ }^{1}$ may be the reason for this more complicated low-temperature motion.

\section{CONCLUSION}

The dynamics in C24 and squalane monolayer films adsorbed on a graphite substrate has been investigated by neutron scattering using the HFBS and DCS instruments located at NIST and by MD simulations. Our focus has been to investigate the effect of the methyl side groups in squalane on its monolayer dynamics.

In general, our MD simulations indicate that the translational diffusive motion in the $\mathrm{C} 24$ and squalane monolayers adsorbed on a graphite basal-plane surface is too rapid to be observed on either the DCS or HFBS instrument in the temperature range investigated. At low temperatures, the nearly dispersionless quasielastic scattering is contributed primarily by the uniaxial rotation of the molecules about their long axis. This rotational motion occurs on time scales accessible to both DCS and HFBS. However, the rotation is not purely uniaxial for squalane due to the presence of its methyl side groups.

As the temperature increases, we identify a contribution to the quasielastic scattering from conformational changes of the molecules associated with the creation and annihilation of gauche defects. These conformational changes are slow enough to be observable on the HFBS just below the monolayer melting point in the case of the $\mathrm{C} 24$ monolayer and at somewhat lower temperatures for the squalane monolayer where the methyl side groups are involved.

Overall, we found that the dynamics in the two monolayer films are qualitatively similar, with only small quantitative differences that could be understood in terms of different masses and moments of inertia of the two molecules. Combined with our findings about the structures of the two systems, ${ }^{1}$ we may conclude that the monolayer films of $\mathrm{C} 24$ and squalane are quite similar both in structure and dynamics. Thus, there is no reason to believe that the branched alkane will be a better lubricant than an $n$-alkane on the nanoscale as opposed to the case in bulk. 


\section{ACKNOWLEDGMENTS}

This work was supported by the U.S. National Science Foundation under Grant Nos. DMR-0109057 and DMR0411748 and by the U.S. Department of Energy through Grant No. DE-FG02-01ER45912. The neutron scattering facilities in this work are supported in part by the National Science Foundation under Agreement No. DMR-0454672. One of the authors (A.D.E.) thanks the Oticon Foundation, Denmark for financial support.

${ }^{1}$ A. D. Enevoldsen, F. Y. Hansen, A. Diama, L. Criswell, and H. Taub, J. Chem. Phys. 126, 104703 (2007), preceding paper.

${ }^{2}$ J. Denis, J. Synth. Lub. 1, 210 (1984).

${ }^{3}$ J. D. Moore, S. T. Cui, H. D. Cochran, and P. T. Crummings, J. Chem. Phys. 113, 8833 (2000).

${ }^{4}$ F. Y. Hansen, L. Criswell, D. Fuhrmann, K. W. Herwig, A. Diama, R. M. Dimeo, D. A. Neumann, U. G. Volkmann, and H. Taub, Phys. Rev. Lett. 92, 461031 (2004).

${ }^{5}$ W. Marshall and S. W. Lovesey, Theory of Thermal Neutron Scattering (Oxford University Press, Oxford, 1971).

${ }^{6}$ S. K. Wang, J. C. Newton, R. Wang, H. Taub, J. R. Dennison, and H. Shechter, Phys. Rev. B 39, 10331 (1989).
${ }^{7}$ M. Abramowitz and I. A. Stegun, Handbook of Mathematical Functions (Dover, New York, 1970).

${ }^{8}$ A. Diama, Ph.D. thesis, University of Missouri-Columbia, 2006.

${ }^{9}$ T. Springer, Quasielastic Neutron Scattering for the Investigation of Diffusive Motions in Solids and Liquids, Springer Tracts in Modern Physics Vol. 64 (Springer-Verlag, Berlin, 1972).

${ }^{10}$ M. J. Bee, Quasielastic Neutron Scattering (Hilger, Bristol, 1988).

${ }^{11}$ F. Y. Hansen and H. Taub, AIP Conf. Proc. 708, 233 (2004).

${ }^{12}$ H. Taub, F. Y. Hansen, L. Criswell, D. Fuhrmann, K. W. Herwig, A. Diama, H. Mo, R. M. Dimeo, D. A. Neumann, and U. G. Volkmann, AIP Conf. Proc. 708, 201 (2004).

${ }^{13}$ J. R. D. Copley and J. C. Cook, Chem. Phys. 292, 477 (2003).

${ }^{14}$ J. R. D. Copley and T. J. Udovic, J. Res. Natl. Inst. Stand. Technol. 98, 71 (1993).

${ }^{15}$ NIST Center for Neutron Research, disk chopper time-of-flight spectrometer, http://www.ncnr.nist.gov/instruments/des/

${ }^{16}$ A. Mayer, R. Dimeo, P. Gehring, and D. Neumann, Rev. Sci. Instrum. 74, 2759 (2003).

${ }^{17}$ NIST Center for Neutron Research, high flux backscattering spectrometer, http://www.ncnr.nist.gov/instruments/hfbs/

${ }^{18}$ K. W. Herwig, B. Matthies, and H. Taub, Mater. Res. Soc. Symp. Proc. 376, 757 (1994).

${ }^{19} \mathrm{http} / / / \mathrm{www} \cdot n$ nenr.nist.gov/dave/

${ }^{20}$ B. Matthies, K. W. Herwig, L. Criswell, A. Diama, H. Mo, H. Taub, and F. Y. Hansen (in preparation).

${ }^{21}$ F. Y. Hansen, K. W. Herwig, B. Matthies, and H. Taub, Phys. Rev. Lett. 83, 2362 (1999). 